\title{
High Quality Muon Detection for the SSC
}

W.X. Gao, J. Li, Z.P. Mao, Y.T. Xu, W.G. Yan, Y. Yang, M.H. Ye, Z.P. Zheng, H.S. Zhou and Y.C. Zhu Institute of High Energy Physics, Beijing 100039 China

M. Atiya, T. Kycia and M. Tannenbaum Brookhaven National Laboratory, Upton, LI, NY 11979

R. Breedon, Winston Ko, $\ddot{R}$. Lander and P. Yager University of California at Davis, Davis, CA 95616

$$
\text { D. Winn }
$$

Fairfield University, Fairfield, CT 06490

H. Hirabayashi and K. Tsuchiya

KEK, National Laboratory for High Energy Physics, Ibaraki 905 Japan

J.S. Kang

Korea University, Seoul 196-701 Korea

R. McNeil

Louisiana State University, Baton Rouge, LA 70803

C. Bromberg

Michigan State University, East Lansing, MI 48824

R. Kass

The Ohio State University, Columbus, OH 43210

Q. Fan, G. Fanourakis, T. Ferbel, S. Kanda, S.L. Olsen,

P.F. Slattery, K. Ueno and M. Zielinski

University of Rochester, Rochester, NY 14627

S.K. Kim and S.R. Schnetzer

Rutgers University, Piscataway, NJ 08854

W. Vernon

University of California at San Diego, La Jolla CA 92093

C. Rosenfeld and J. Wilson

University of South Carolina, Columbia, SC 29208

M. Mohammadi

State University of New York at Stony Brook, Stony Brook, NY 11794

C.A. Fry and F. Bird

Superconducting Supercollider Laboratory, Dallas, TX 75237

J. Ritchie and A. Yamashita

. University of Texas, Austin, TX 78712

A. Abashian, K. Gotow, N. Morgan and L. Piilonen

Virginia Polytechnic Institute and State University, Blacksburg, VA 24061

P. Cushman, S. Hou and V. Singh

Yale University, New Haven, CT 06511

UNIVERSITY OF ROCHESTER APPROVED FOR RELEASE OR
PUBICATION - O.R. PATEMT GROUP PUB UYGATION - O.R. PATENTT GROUP 


\section{DISCLAIMER}

This report was prepared as an account of work sponsored by an agency of the United States Government. Neither the United States Government nor any agency thereof, nor any of their employees, make any warranty, express or implied, or assumes any legal liability or responsibility for the accuracy, completeness, or usefulness of any information, apparatus, product, or process disclosed, or represents that its use would not infringe privately owned rights. Reference herein to any specific commercial product, process, or service by trade name, trademark, manufacturer, or otherwise does not necessarily constitute or imply its endorsement, recommendation, or favoring by the United States Government or any agency thereof. The views and opinions of authors expressed herein do not necessarily state or reflect those of the United States Government or any agency thereof. 


\section{DISCLAIMER}

Portions of this document may be illegible in electronic image products. Images are produced from the best available original document. 


\section{High Quality Muon Detection for the SSC}

W.X. Gao, J. Li, Z.P. Mao, Y.T. Xu, W.G. Yan, Y. Yang, M.H. Ye, Z.P. Zheng, H.S. Zhou and Y.C. Zhu Institute of High Energy Physics, Beijing 100039 China

M. Atiya, T. Kycia and M. Tannenbaum Brookhaven National Laboratory, Upton, LI, NY 11973

R. Breedon, Winston Ko, R. Lander and P. Yager

University of California at Davis, Davis, CA 95616

- D. Winn

Fairfield University, Fairfield, CT 06430

H. Hirabayashi and K. Tsuchiya

KEK, National Laboratory for High Energy Physics, Ibaraki 305 Japan

J.S. Kang

Korea University, Seoul 136-701 Korea

R. McNeil

Louisiana State University, Baton Rouge, LA 70803

C. Bromberg

Michigan State University, East Lansing, MI 48824

R. Kass

The Ohio State University, Columbus, OH 43210

Q. Fan, G. Fanourakis, T. Ferbel, S. Kanda, S.L. Olsen, P.F. Slattery, K. Ueno and M. Zielinski

University of Rochester, Rochester, NY 14627

S.K. Kim and S.R. Schnetzer

Rutgers University, Piscataway, NJ 08854

W. Vernon

University of California at San Diego, La Jolla CA 92093

C. Rosenfeld and J. Wilson

University of South Carolina, Columbia, SC 29208

M. Mohammadi

State University of New York at Stony Brook, Stony Brook, NY 11794

C.A. Fry and F. Bird

Superconducting Supercollider Laboratory, Dallas, TX 75237

J. Ritchie and A. Yamashita

University of Texas, Austin, TX 78712

A. Abashian, K. Gotow, N. Morgan and L. Piilonen

Virginia Polytechnic Institute and State University, Blacksburg, VA 24061

P. Cushman, S. Hou and V. Singh

Yale University, New Haven, CT 06511 


\section{Contents}

1 Introduction $\quad 3$

1.1 Review of the $L^{*} / G E M$ Scheme for Muon Measurements. . . . . . . . . . 3

1.2 The Proposed High Field Option . . . . . . . . . . . . . 4

1.2.1 Point-Slope Measurements of Muon Momenta ............ 4

1.2.2 Forward-Backward Muon Coverage . . . . . . . . . . . 9

1.2 .3 "Robustness" ....................... 10

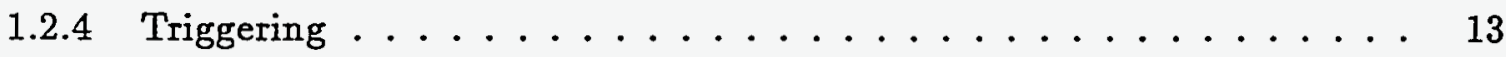

1.2.5 Effects on the Rest of the Detector . . . . . . . . . . . 13

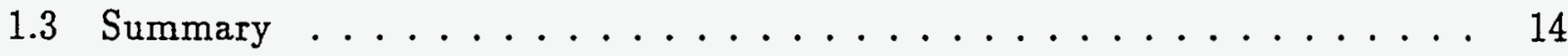

2 Physics, Capabilities of a High Field Detector 16

$2.1 p p \longrightarrow W_{L}{ }^{+} W_{L}{ }^{+} X \ldots \ldots \ldots \ldots \ldots \ldots$

$2.2 H i g g s \longrightarrow Z^{\circ} Z^{*} \longrightarrow \mu^{+} \mu^{-} \mu^{+} \mu^{-} \ldots \ldots \ldots \ldots \ldots \ldots$

2.3 The Effect of the Field on Jet Detection . . . . . . . . . . . 19

2.4 The Effect of the Field on Isolation Cuts . . . . . . . . . . . . 20

3 Muon Detection Simulation $\quad 21$

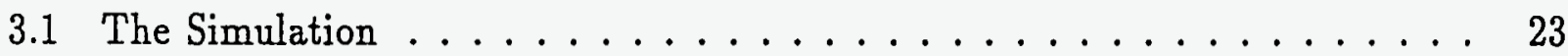

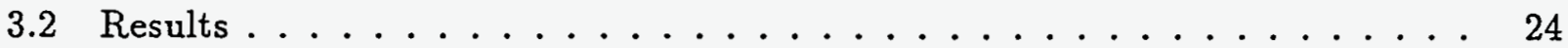

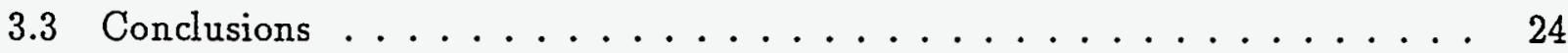

4 Magnet and Mechanical Support 26

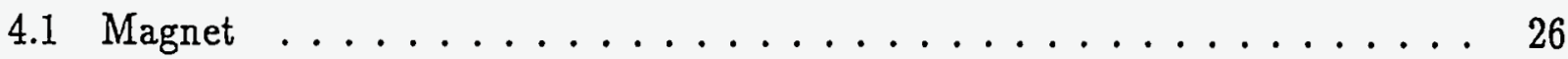

4.2 Mechanical Assembly and Support ................. 27

5 Effects of the High Field on the elements of the Inner Detector $\quad 30$

5.1 The Effect of the Field on the Inner Tracker . . . . . . . . . . . . 30

5.2 The Effect of the Field on the Endcap Region of the Middle Tracker . . . . 32

5.3 The Effect of the Field on Calorimetry ... . . . . . . . . . 32

6 Conclusions $\quad 33$ 


\begin{abstract}
We present an alternative approach for muon detection at the SSC based on a high field magnet ( $B=5$ Tesla) of "moderate" dimensions (radius $=3.5 \mathrm{~m}$; overall length $=15$ meters). A precision determination of a muon's momentum is made using a point-slope method, where the point is the interaction point and the slope is the direction of the muon's track as it exits the magnet coil. Momentum resolutions on the order of $\Delta p_{t} / p_{t} \simeq 4 \%$ are possible over large solid angles for muon momenta as high as $1 \mathrm{TeV}$ with rather modest alignment $(\sim 200 \mu \mathrm{m})$ tolerances, with standard drift chamber resolutions $(\sim 100 \mu \mathrm{m})$, and with no reliance on the performance of an inner tracking system. Robustness is provided by additional momentum measurements in the inner tracker and in a solid iron spectrometer utilizing the magnet's return yoke. The total cost of the system, including proper magnetic shielding for the magnet and a forward-backward iron-core toroid muon detection system with $\Delta p / p \simeq 9 \%$, is comparable to that of the low-field $L^{*} /$ GEM approach that does not provide a magnetic flux return nor a forward-backward muon system. The magnet leaves the same amount of space for inner detector items as does the large, low-field $L^{*}$-type system, while providing a substantially higher magnetic field in the inner tracking volume.
\end{abstract}




\section{Introduction}

\subsection{Review of the $L^{*} /$ GEM Scheme for Muon Measurements.}

The central theme of the $\mathrm{L}^{*} / \mathrm{GEM}$ detector design is its emphasis on precise measurements on gammas, electrons and muons. The detector concept, as presented in the group's Expression of Interest (EOI) [1], is dominated by a large (inner radius $=8.5 \mathrm{~m}$; overall length $=28 \mathrm{~m})$, relatively low-field $(B=0.8$ Tesla) solenoid magnet that supports an air-core muon spectrometer situated radially outside of an inner tracker system and electromagnetic and hadronic calorimeters. All detection elements are inside the coil. The muon spectrometer, which extends radially from $R=3.6 \mathrm{~m}$ to $8.5 \mathrm{~m}$, determines muon momentum through a measurement of the sagitta of the muon's trajectory in the solenoid's magnetic field (see Fig. 1). The alignment tolerances of the muon spectrometer system are controlled by the requirement of a precise measurement of the trajectory's sagitta $\delta$, where

$$
\delta(\mathrm{mm})=\frac{0.3 B(\mathrm{Tesla}) L^{2}(\mathrm{~m})}{8 p_{t}(\mathrm{TeV} / \mathrm{c})} \simeq \frac{0.75 \mathrm{~mm}}{p_{t}(\mathrm{TeV} / \mathrm{c})} .
$$

Thus, a $\Delta p_{t} / p_{t} \simeq 5 \%$ measurement at $p_{t}=0.5 \mathrm{TeV} / \mathrm{c}$ requires $\sim 75 \mu \mathrm{m}$ resolution on the sagitta measurement, placing strict requirements on the alignment of the various elements in the muon spectrometer. These precise alignment tolerances for large chambers separated by large distances $(\Delta R \sim 5 \mathrm{~m})$ result in a muon system that accounts for the largest part of the estimated cost for the $\mathrm{L}^{*} / \mathrm{GEM}$ detector.

While the proposed muon spectrometer gives the desired $5 \%$ resolution (at $p_{t}=0.5 \mathrm{TeV} / \mathrm{c}$ ) over the central region of the detector (i.e., for $|\eta|<1.4$ ), the resolution for larger $|\eta|$ values deteriorates rapidly, as can be seen in Fig. 2. Improvements in the resolution for the forward directions will require systems that cover very large areas. The $L^{*} / G E M$ magnet provides a rather modest field of only $0.8 \mathrm{~T}$ in the inner tracking region. As a result, the EOI projects a momentum resolution for the inner tracker of $\Delta p / p \simeq 130 \% p(\mathrm{TeV} / \mathrm{c})$, which precludes, for example, the ability to measure electron charges reliably for momenta above $\sim 200 \mathrm{GeV} / \mathrm{c}$. Moreover, the size of the magnet makes the provision of a proper path for the returning magnetic flux difficult. Three approaches for dealing with the magnet's return field have been suggested:

1. a passive-iron return path, which would require approximately 25,000 metric tons of iron and an increase of $\sim 2 \mathrm{~m}$ in the radial dimension of the detector;

2. a second superconducting coil of even larger radius, whose sole function would be to return the field;

3. no flux return at all.

The first two approaches add substantially to the cost of the detector and interaction hall 


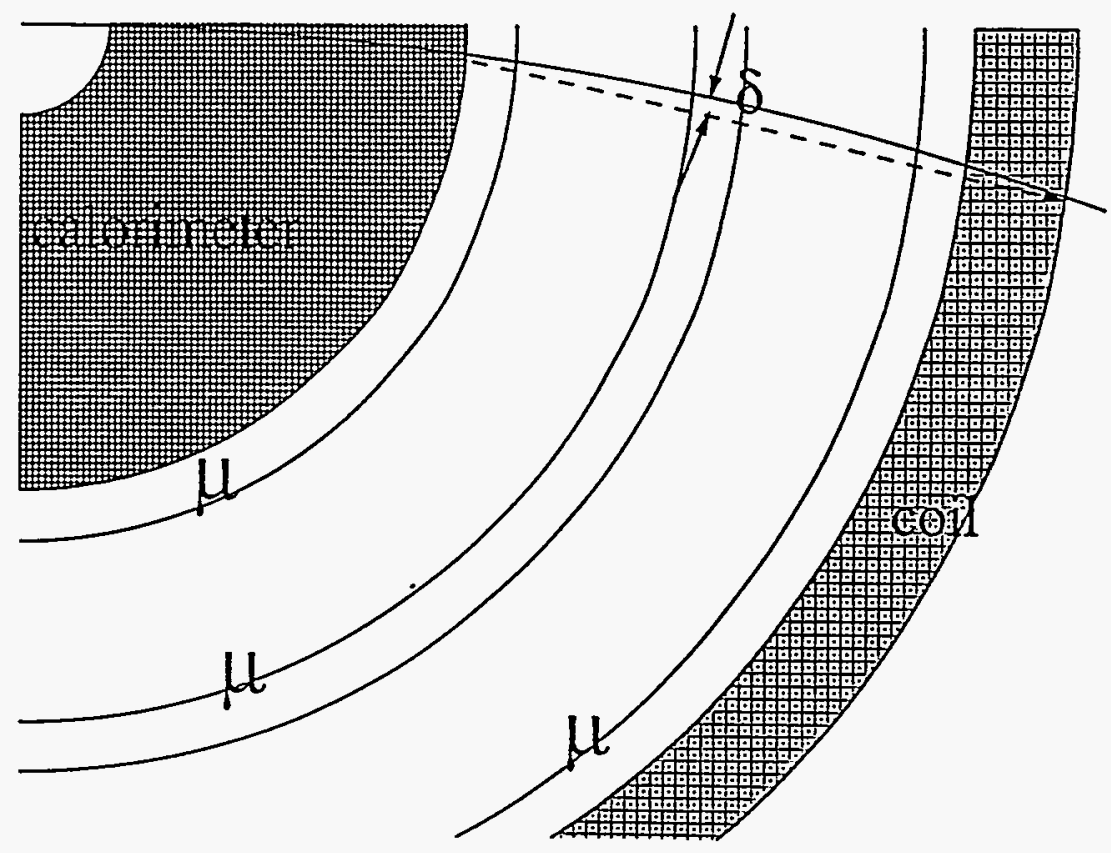

Figure 1: A cross section of the $\mathrm{L}^{*} /$ GEM detector illustrating the sagitta technique used for muon momentum measurements.

( $\Delta$ cost $\geq \$ 50 \mathrm{M})$, and the last approach results in significant magnetic fields that extend well above ground level near the position of the detector.

\subsection{The Proposed High Field Option}

In light of the limited $\eta$ coverage for muons, the compromised momentum resolution of the inner tracking system, and the flux return concerns of the GEM detector arrangement, we propose an alternative approach to muon detection, one that emphasizes excellent resolution for very high momenta over a larger solid angle, and is based on detection elements with alignment tolerances that are an order-of-magnitude less demanding than that of the EOI concept.

Our proposed system, illustrated in Fig. 3, is based on a high-field ( $B=5$ Tesla) magnet with dimensions (inner radius $=3.5 \mathrm{~m}$, overall length $=15 \mathrm{~m}$ ) selected to have minimal interference with the other detection systems. We estimate that the cost of our proposed system, which includes a properly shielded magnet and iron-core-toroid muon spectrometers covering the forward-backward regions $(|\eta| \leq 3.5)$, is comparable to that of the $\mathrm{L}^{*} / \mathrm{GEM}$ system.

\subsubsection{Point-Slope Measurements of Muon Momenta}

In the high-field scheme under discussion here, we abandon the reliance on a sagitta measurement for the determination of muon momenta and employ instead a point-slope measurement [2]. For the point, we exploit the precisely known transverse position of the interaction point; for the slope, we use the angle of the muon's trajectory (relative to the radial direction) as 


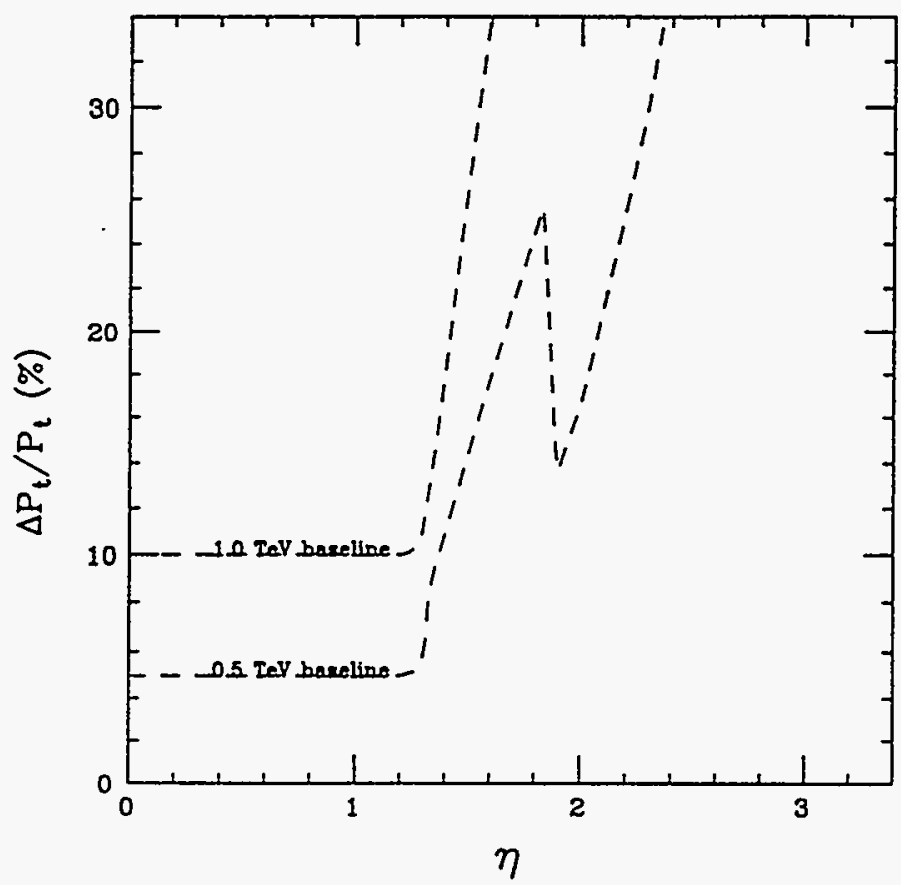

Figure 2: $\Delta p_{t} / p_{t} v s|\eta|$ for the $L^{*} /$ GEM detector.

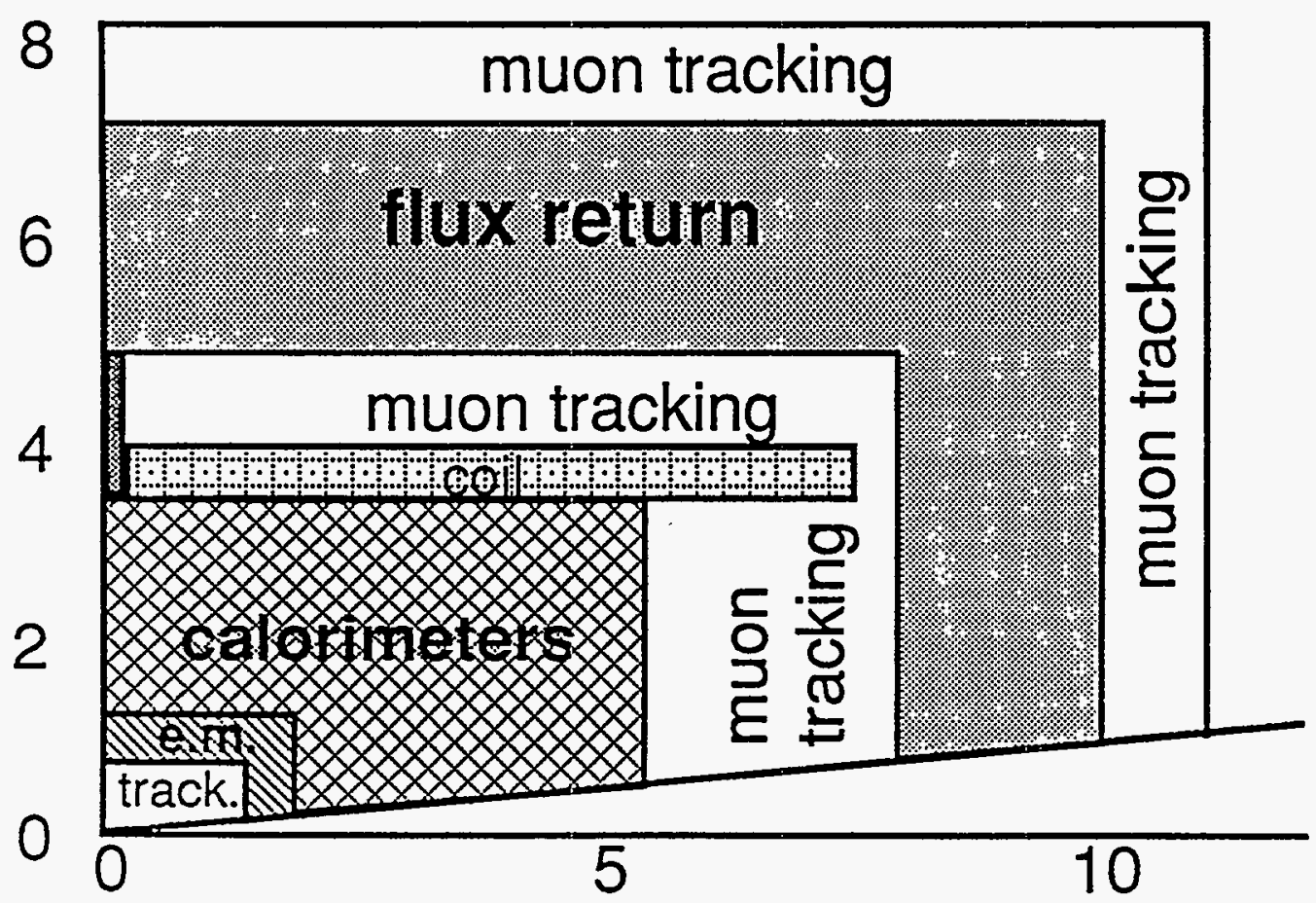

Figure 3: The proposed high field detector arrangement. 


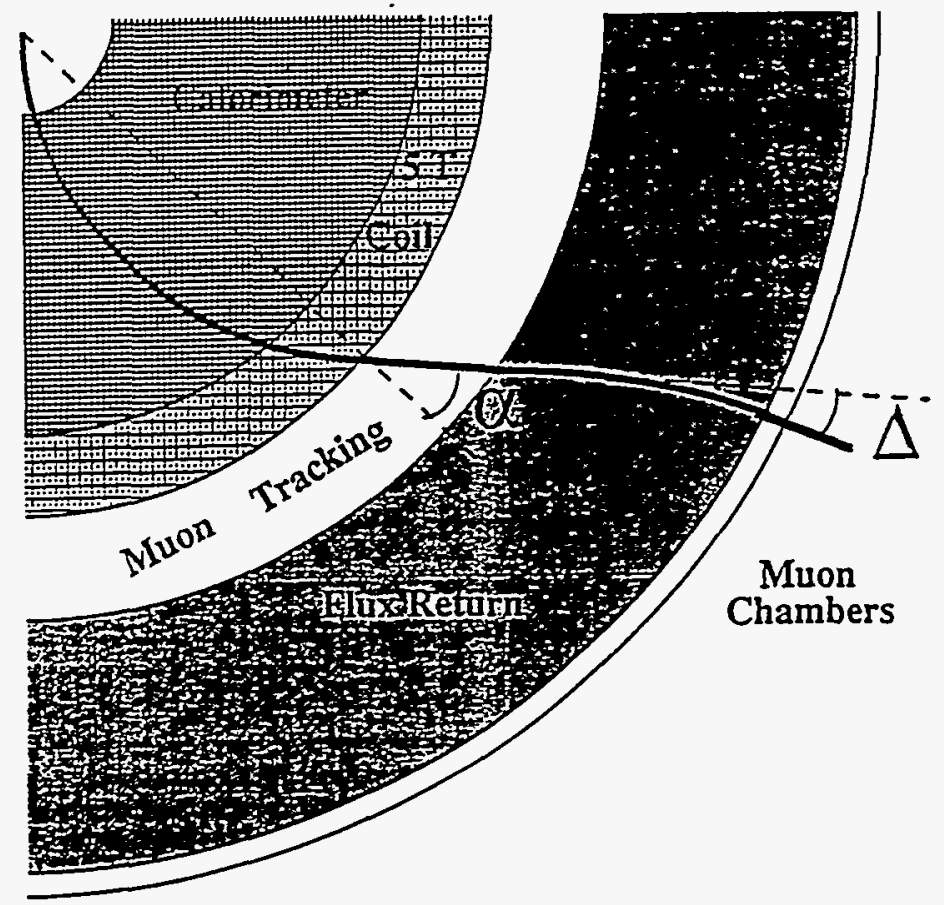

Figure 4: A cross section view of the proposed system illustrating the technique used for muon momentum measurements. The angle $\alpha$ is inversely proportional to the muon's transverse momentum.

it emerges from a solenoid coil situated radially outside of the inner detector, as illustrated in Fig. 4. We estimate that a $15 \mathrm{~m}$-long superconducting coil with inner radius $R=3.5 \mathrm{~m}$ (about the same as the inner radius of the inner muon chambers of the $L^{*} / G E M$ design) can have a field of $B=5$ Tesla for approximately the same cost as the large (28 m-long, $R=8.5 \mathrm{~m}$ ) low-field ( $B=0.8$ Tesla) magnet described in the GEM group's EOI. A magnet coil of this size leaves the dimensions of the inner detector essentially unchanged.

The momentum of the muon can be directly inferred from the angle between the track vector and the radial direction (the angle denoted as $\alpha$ in Fig. 4) as:

$$
\alpha(\mathrm{mrad})=\frac{0.3 B(\mathrm{Tesla}) R(\mathrm{~m})}{2 p_{t}(\mathrm{TeV} / \mathrm{c})} \simeq \frac{2.7 \mathrm{mrad}}{p_{t}(\mathrm{TeV} / \mathrm{c})}
$$

The fundamental limit on the precision of this measurement derives from the multiple scattering of the muons in the material of the inner detector, given by the rms multiple-scattering angle $\theta_{m e}$, where

$$
\theta_{m s} \simeq \frac{0.014(\mathrm{GeV})}{p} \sqrt{\frac{x}{x_{r l}}} .
$$

Here, $x / x_{r l}$ is the amount of material in the inner detector and magnet coil in units of radiation lengths. For the inner detector, we take the the amount of material at $\theta=90^{\circ}$ to be $x / x_{r l} \simeq 130$, which allows for a $30 x_{r l}$ EM calorimeter, an $8 \lambda\left(\simeq 80 x_{r l}\right)$ stainless-steel radiator hadron calorimeter and a $20 x_{r l}$ "thick" superconducting coil and cryostat. In this configuration, the multiple scattering uncertainty is

$$
\theta_{m s} \simeq \frac{0.16 m r a d}{p_{t}(T e V)}
$$




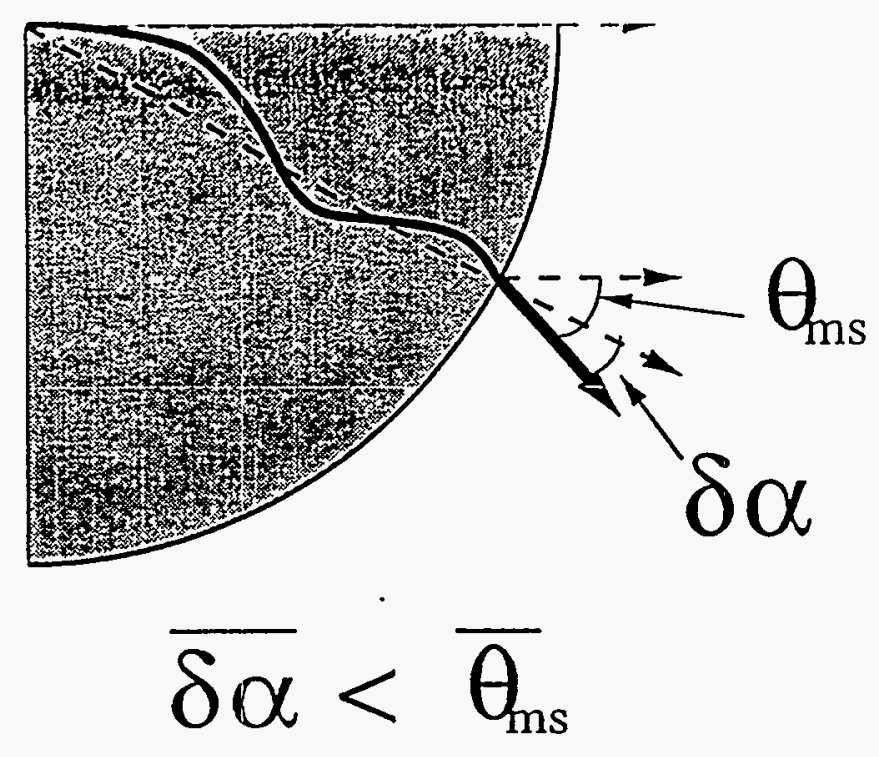

Figure 5: An illustration of the correlation between the multiple scattering angle $\theta_{m s}$ and the error in the radial direction caused by the multiple scattering induced transverse displacement of the trajectory. The resulting error in $\alpha$ is smaller than $\theta_{m s}$.

and a simple estimate of the limiting precision on $\Delta p_{t} / p_{t}$ is given by

$$
\frac{\Delta p_{t}}{p_{t}} \simeq \frac{\theta_{m s}}{\alpha}=0.06,
$$

which (at $\theta=90^{\circ}$ ) is independent of $p_{t}$. In fact, a more precise calculation indicates that the situation is somewhat better than this. Multiple scattering, in addition to introducing an uncertainty in the angle $\alpha$, produces a transverse displacement that is correlated with the multiple scattering angle. This translation results in a change in the inferred radial direction that partially compensates the uncertainty in $\alpha$, as illustrated in Fig. 5. The $\alpha$ distribution for a complete GEANT simulation of a sample of $1 \mathrm{TeV} / \mathrm{c}$ muon tracks, including, plural- as well as multiple-scattering, is shown in Fig. 6 . Here the multiple-scattering limit on muon momentum resolution is just the rms width of the distribution, which is $\Delta p_{t} / p_{t}=4.1 \%$. Simulations for $100 \mathrm{GeV} / \mathrm{c}$ muon tracks yield the same multiple scattering limit on the resolution.

The multiple scattering limit sets the scale of precision required for measurementsfor measurements up to $p_{t} \sim 1 \mathrm{Te} / \mathrm{V} / \mathrm{c}$, the angle of the track exiting the coil must be measured with a precision that is $\leq 0.1 \mathrm{mrad}$, This can be readily done in a radial space of $\Delta R \sim 1 \mathrm{~m}$ using conventional tracking systems. The track's exit position from the coil relative to the interaction point is used to define the radial direction and must be known with a precision of $<350 \mu \mathrm{m}$. This sets the scale for relative alignment requirements. Thus, systems with conventional alignment tolerances will provide an excellent momentum resolution of $\Delta p_{t} / p_{t} \simeq 5 \%$ for $p_{t}$ up to $\sim 1 \mathrm{TeV} / \mathrm{c}$ ! (For $p_{t}$ values above $1 \mathrm{TeV} / \mathrm{c}$, the $\sim 0.1 \mathrm{mrad}$ measuring precision results in a gradual deterioration in $\Delta p / p$.) The above discussion is for $\theta=90^{\circ}$. The results of an analysis of our proposed system extended over all angles and for a variety of muon momenta are shown in Fig. 7. Also shown are the expectations for the $L^{*} /$ GEM design.

(If, in addition to the external measurements, a single position measurement, with a 


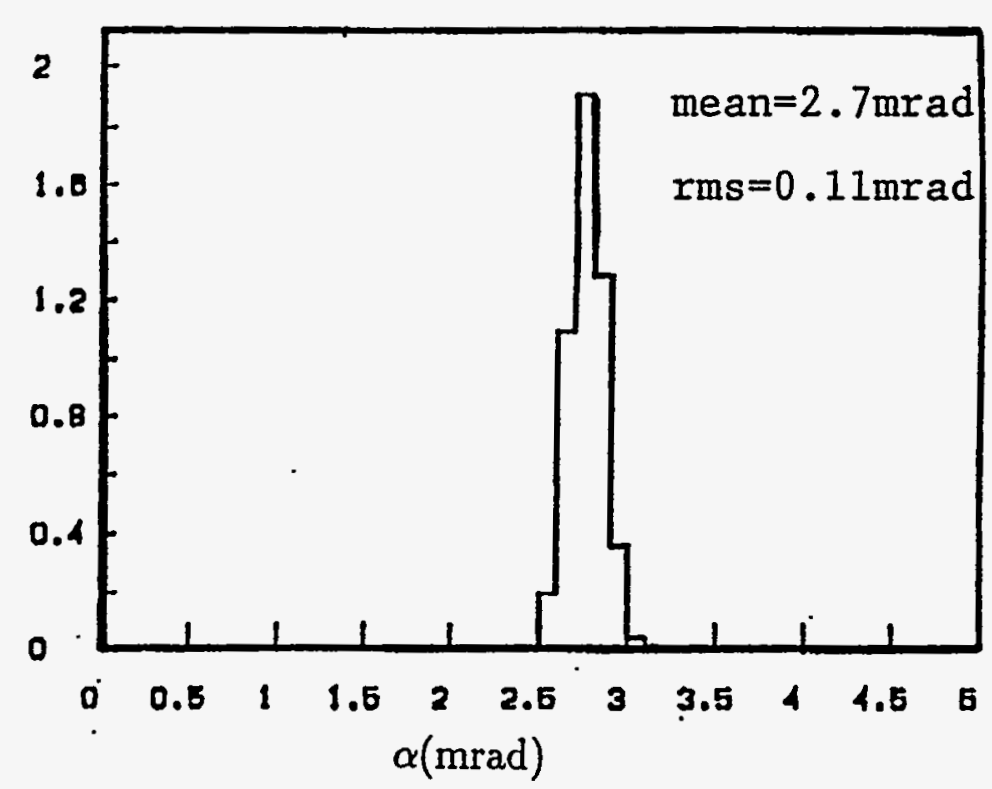

Figure 6: The distribution on $\alpha$ from a simulation of $1 \mathrm{TeV} / \mathrm{c}$ muons. The rms spread is 0.11 mrad.

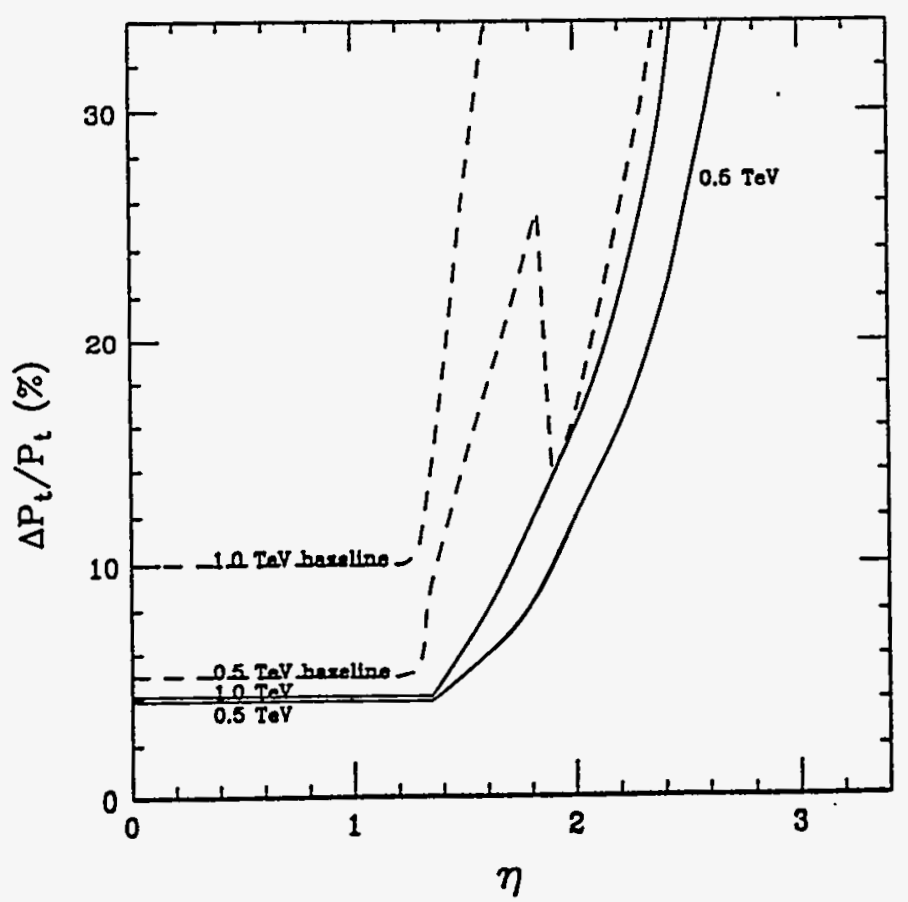

Figure 7: The expected transverse momentum resolution $\left(\Delta p_{t} / p_{t}\right)$ for the proposed system from measurements in the middle tracker only. 


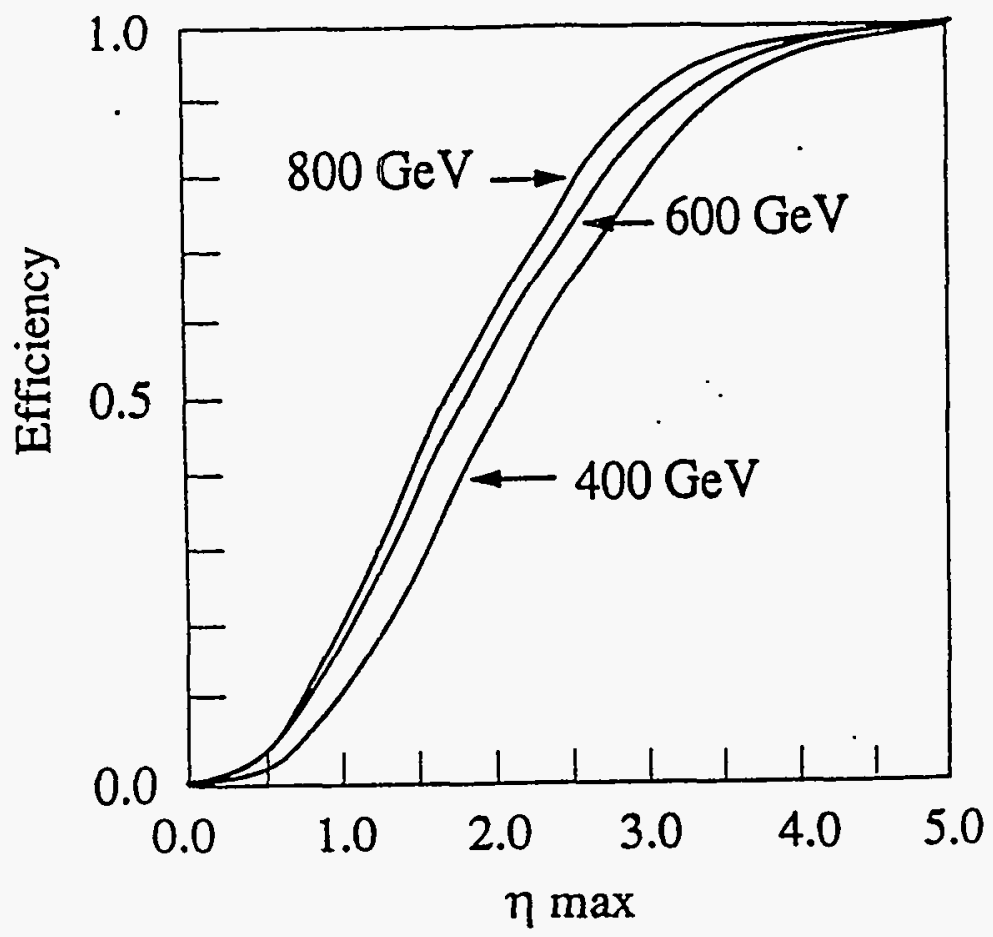

Figure 8: The efficiency for Higgs $\longrightarrow \mu^{+} \mu^{-} \mu^{+} \mu^{-}$vs the maximum $|\eta|$ of the muon acceptance (from ref. 3).

spatial precision of $\sigma \sim 50 \mu \mathrm{m}$, is used from the outer region of the inner tracker, the resolution can be further improved to the $\sim 3 \%$ level. Of course, if the complete track is found in the inner tracker, a totally independent momentum measurement can be made and used to improve the overall resolution.)

\subsubsection{Forward-Backward Muon Coverage}

If one is to attain the physics goals of a high-p $p_{t}$ detector at the SSC, momentum resolutions must not deteriorate at $|\eta| \sim 1.5$, as it does in Fig. 7. For example, Fig. 8 shows the efficiency for detecting all four muons from the reaction

$$
p p \longrightarrow \text { Higgs }+X ; \quad \text { Higgs } \longrightarrow Z^{\circ} Z^{\circ} \longrightarrow \mu^{+} \mu^{-} \mu^{+} \mu^{-},
$$

for different Higgs masses as a function of the maximum $|\eta|$ value of the muon acceptance [3]. From the figure it is clear that there is a premium on having good muon detection down to $|\eta|=3 \sim 3.5$, particularly for lighter Higgs' masses; if one is restricted to $|\eta| \leq 1.4$, the efficiency for this reaction is limited. (This is particularly true for Higgs masses below $2 M_{Z}$, where the Higgs $\longrightarrow Z^{\circ} Z^{*}$ decay channel is important. Here, since the Higgs is relatively light, it tends to be produced in the forward direction, and, since the branching ratio is small, event rates are small and good efficiency is particularly important.) A discussion of the performance of our proposed high field system for this reaction is given below in Section 2.1 .

To provide adequate momentum resolution in the forward-backward region, we propose 


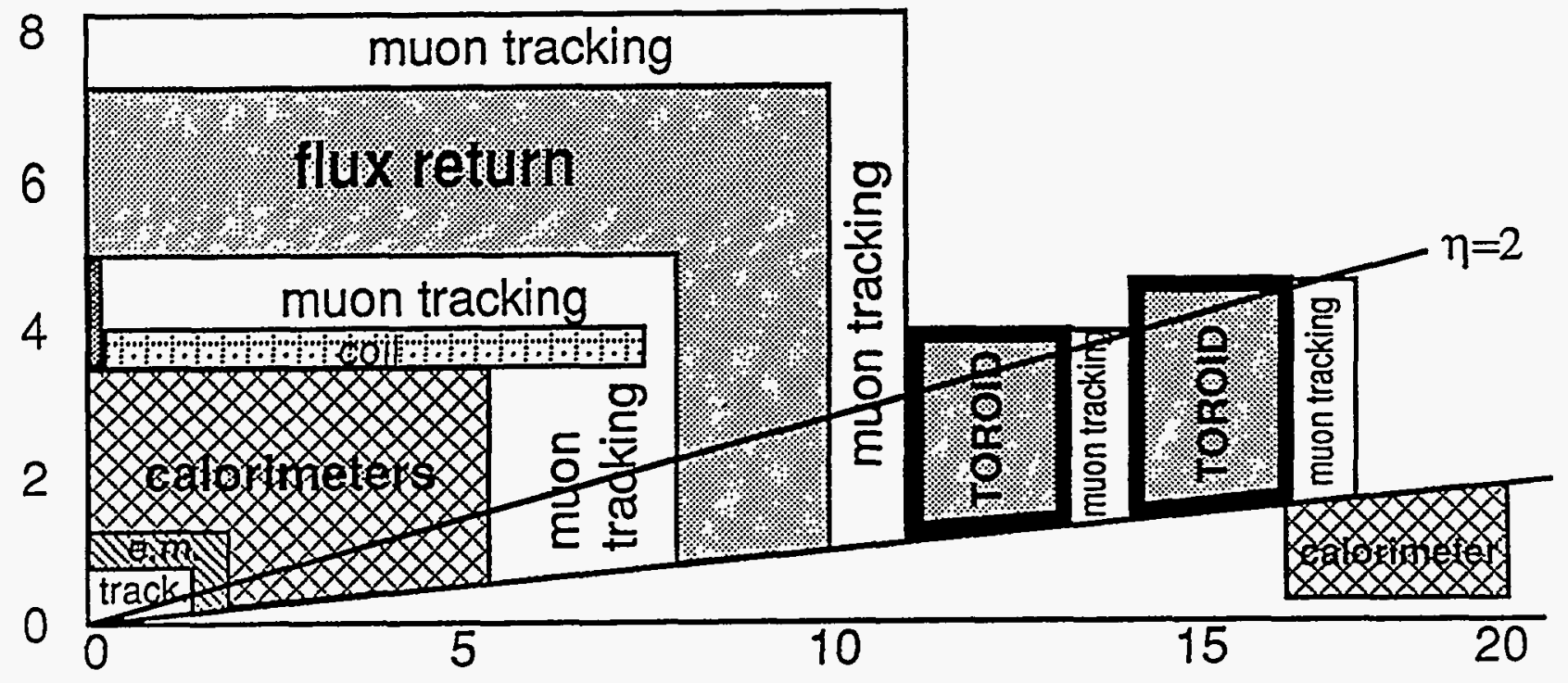

Figure 9: A plan view of the proposed high field system including the forward-backward iron-core toroid systems.

to incorporate iron-core toroids covering the $2 \leq|\eta| \leq 3.5$ region, as indicated in Fig. 9 . Two 2-meter thick toroids at each end of the detector will provide a multiple scattering limited momentum resolution in this region of $\Delta p / p \simeq 9 \%$. Here, the relative compactness of the high field system allows this to be done with $R \simeq 5 \mathrm{~m}$ toroids; each toroid weighs about 1.2 ktons. With this system, we get reasonable muon coverage out to $|\eta| \leq 3.5$. Figure 10 shows $\Delta p_{t} / p_{t} v s|\eta|$ for the complete system.

\subsection{3 "Robustness"}

The measurement of a single, straight-line track segment by itself would hardly qualify as a "robust" measurement. A low-momentum track could scatter through a large angle and emerge from the coil near the radial direction, faking a high momentum track. Therefore, the track segment measurement must be tightly constrained by other measurements, both at the trigger level and beyond. In our proposed system, we use the measurement outside the coil to infer the position of hits both in the inner-tracking system and in a second outer-tracking system located behind the iron flux return of the magnet.

Comparison with Measurements in the Inner Tracker The track coordinates and momentum determined in the tracking system just outside of the coil (which is subsequently referred to as the "middle tracker") can be used to extrapolate to the inner tracking device and correlated with measurements of the same track there. This is similar to the constraint used in the $L^{*} / G E M$ design. (This is the only constraint in the $L^{*} / G E M$ system.) All things being equal, the comparison in the high-field system is more constraining because the higher magnetic field will yield a better momentum resolution in the inner tracker. 


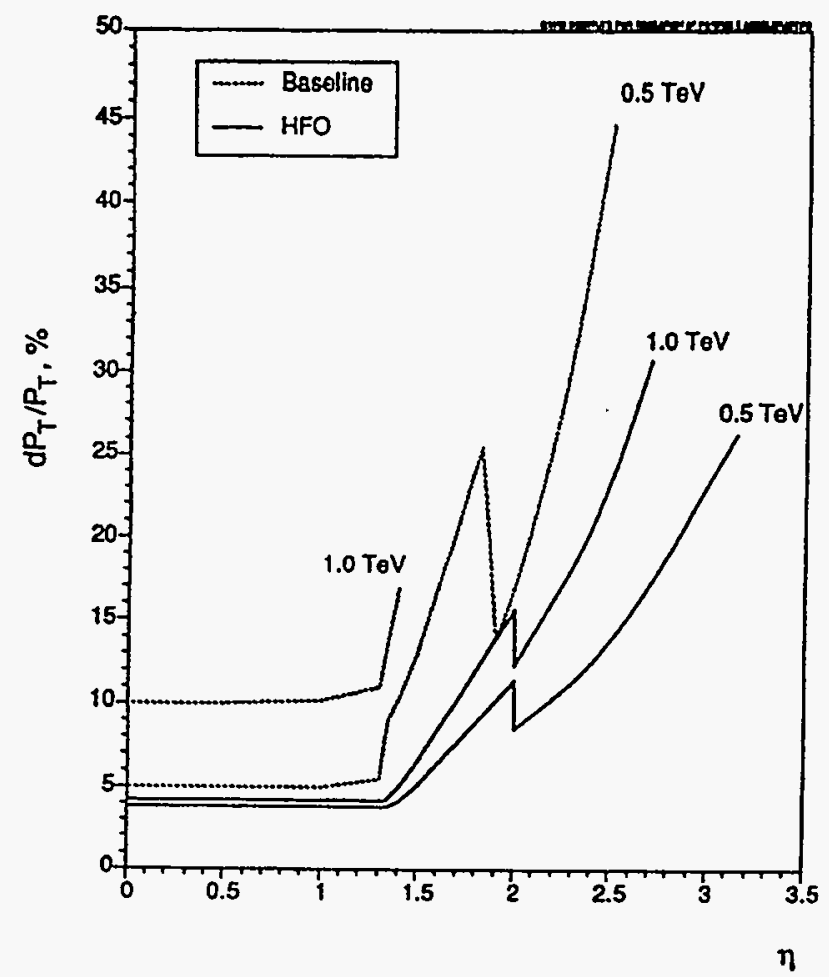

Figure 10: $\Delta p_{t} / p_{t} v s|\eta|$ for the complete system, including the forward-backward iron toroid system.

Comparison with Measurements in the Outer Tracker At very high luminosities (e.g., in excess of $10^{34} \mathrm{~cm}^{-2} \mathrm{~s}^{-1}$ ) the performance of the inner tracking device may be compromised. Therefore, we place our emphasis on constraints that are independent of the inner tracking system.

In our proposed system, the magnetic flux produced by the superconducting coil is returned through an iron yoke with a radial thickness of $\Delta R=2.25 \mathrm{~m}$, as shown in Fig. 4 . The magnetic field in the iron is around 2 Tesla and is approximately perpendicular to radial particle trajectories for $|\eta|<1.75\left(\theta>20^{\circ}\right)$. Thus the flux return is well suited for use as the bending element of a second muon spectrometer. A tracking system situated outside of the return yoke (the "outer tracker") measures the exit position and slope of the muon's trajectory as it exits the iron.

Measurements of a muon's position and angle in the middle tracker uniquely predict the position and slope of the track when it emerges from the iron return yoke. A high momentum muon will emerge from the iron yoke very near the radial direction; multiple scattering in the $2.25 \mathrm{~m}\left(\simeq 125 x_{r l}\right)$ thick iron smears the exit angles by about $0.15 \mathrm{mrad} / p(\mathrm{TeV} / \mathrm{c})$. The exit position (in the bend plane) of the track from the iron return can be used to make a second determination of the momentum: the difference between the straightline extrapolation of the track in the middle tracker and the measured exit position (i.e., $\Delta$ in Fig. 4 ) is

$$
\Delta(\mathrm{mm})=\frac{0.3 B(\mathrm{Tesla}) L(\mathrm{~m})^{2}}{2 p(\mathrm{TeV} / \mathrm{c})} \simeq \frac{1.9 \mathrm{~mm}}{p_{t}(\mathrm{TeV} / \mathrm{c})}
$$

Multiple scattering smears the exit position of the muon introducing an uncertainty of $\Delta_{m s} \simeq$ $270 \mu \mathrm{m} / p(\mathrm{TeV} / \mathrm{c})$, which limits the resolution of the second momentum measurement. If the 


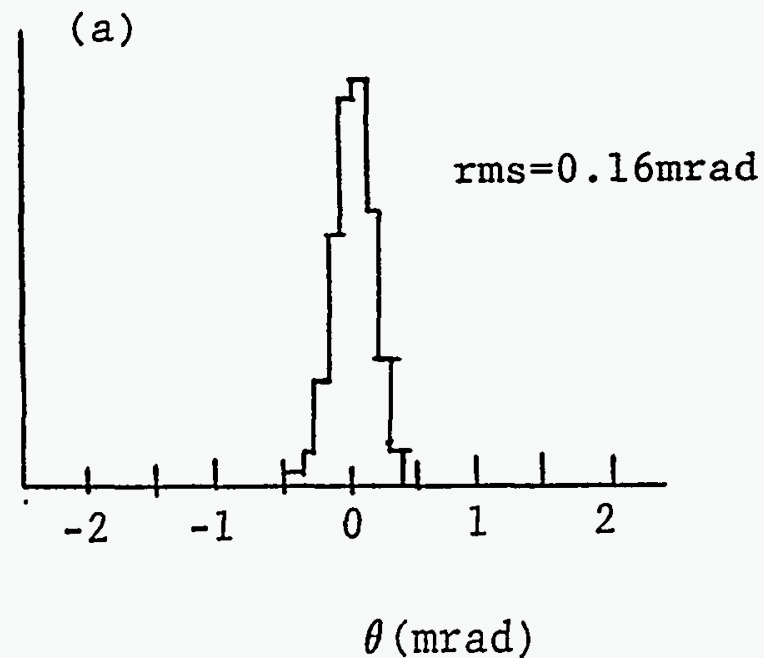

N

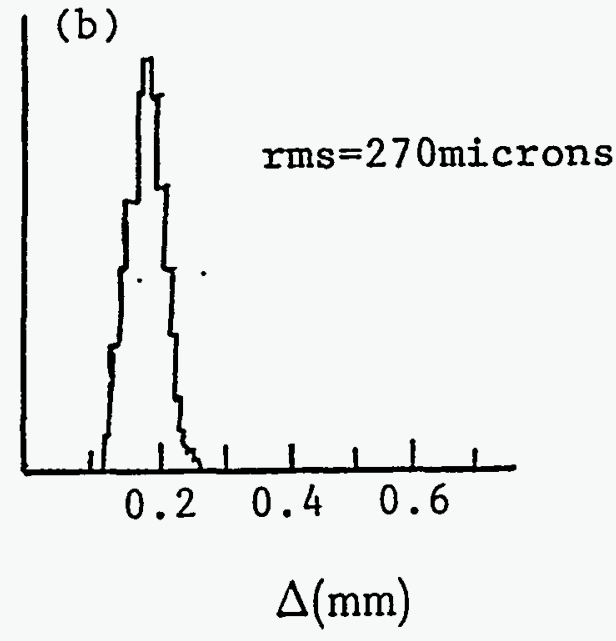

Figure 11: (a) The distribution in angle of $1 \mathrm{TeV} / \mathrm{c}$ muons emerging from the iron return yoke (at $\left.90^{\circ}\right)$. (b) The distribution in the displacement $\Delta$ of the muon's trajectory from its straight line extrapolated position for a sample of $1 \mathrm{TeV}$ muons.

exit position is measured with a precision of $200 \mu \mathrm{m}$, the second momentum measurement will have a resolution of $\Delta p / p \simeq 18 \%$ for $1 \mathrm{TeV} / \mathrm{c}$ muons; for $500 \mathrm{GeV} / \mathrm{c}$ and below, the effect of the measurement error becomes negligible and the resolution is $\simeq 15 \%$. This measurement can be compared with the measurement of the angle $\alpha$ in the middle tracker, providing a powerful constraint. The results of a GEANT simulation for the exit angle and position for 1 $\mathrm{TeV} / \mathrm{c}$ tracks when they emerge from the iron return yoke are shown in Figs. 11 (a) and (b), respectively. The width of the $\Delta$ distribution in Fig. 11 (b) reflects the multiple-scattering limits on the second momentum measurement as discussed above. (The simulation includes single- and plural-scattering.) The results of a simulation of a sample of $p=100 \mathrm{GeV} / \mathrm{c}$ muons produced the expected results with no surprises.

Measurements in the outer tracker are performed in a very quiet environment-the material between the interaction point ( $\sim 25 \lambda$ at $90^{\circ}$ and more at smaller angles) and the outer tracker reduce the particle fluxes in the worst locations to $\sim 1 \mathrm{~Hz} / \mathrm{cm}^{2}$. The requirement that the measured exit position agree with the value predicted by the middle tracker with an rms precision of $270 \mu \mathrm{m}$ provides a powerful (two-dimensional) constraint. While these constraints provide powerful handles for rejecting background, they correspond to rather comfortable precision requirements on the angle ( $\leq 100 \mathrm{mrad})$ and position $(\leq 200 \mu \mathrm{m})$ measurements in the outer tracker. The relative alignment requirements between the middleand outer-tracker are about an order-of-magnitude less demanding than is the case for the relative alignment of the different muon chambers in the $L^{*} / G E M$ system.

In summary, "robustness" is provided by redundant measurements of the track parameters in the inner tracker, the only constraint available to the $\mathrm{L}^{*} / \mathrm{GEM}$ system, and by the correlations with the position and angle of the track as it emerges from the magnetized iron 
return yoke of the solenoid. The latter constraints, which do not obtain in the $\mathrm{L}^{*} / \mathrm{GEM}$ design, will be available in the event of high luminosity running, when the operation of the inner tracker may be severely compromised.

Further questions that relate to robustness, such as the expected particle rate at each detector element, the "corruption" of muon trajectory measurements by accompanying delta rays and electromagnetic "debris," and estimates of the effectiveness of the track constraints are being studied. These studies are discussed in more detail below in Section 3.

\subsubsection{Triggering}

The simplicity of the muon measurement scheme, namely the measurement of straightline track segments in the middle and outer trackers, allows one to generate simple but powerful triggers both at Level-1 and Level-2. The angle $\alpha$ is inversely proportional to the muon's transverse momentum; a requirement that $\alpha \leq 270 \mathrm{mrad}$ corresponds to a transverse momentum selection of $p_{t} \geq 10 \mathrm{GeV} / \mathrm{c}$. Using ISAJET, we estimate that the requirement that $\alpha \leq 270 \mathrm{mrad}$, corresponding to the indication of a particle track in a coarse $(\sim 27 \mathrm{~cm}$ wide) radial road, together with the presence of some hits nearby in the outer tracker provides a Level-1 trigger that is efficient for $p_{t} \geq 10 \mathrm{GeV} / \mathrm{c}$ muons and has a rate of $1 \mathrm{KHz}$ at the SSC design luminosity of $10^{33} \mathrm{~cm}^{-2} \mathrm{~s}^{-1}$. Since this is an order-of-magnitude lower than the rate necessary for a Level-1 trigger at the SSC, the same scheme should be applicable for operation at luminosities of $10^{34} \mathrm{~cm}^{-2} \mathrm{~s}^{-1}$.

For Level-2, the position of the muon track in the outer tracker can be correlated with the extrapolated position from the middle tracker measurement to make a more precise selection of the muon's transverse momentum. A $\pm 5 \mathrm{~cm}$ correspondence is equivalent to a selection of $p_{t}>40 \mathrm{GeV} / \mathrm{c}$, which will have a rate of a few hundred Hertz at a luminosity of $10^{33} \mathrm{~cm}^{-2} \mathrm{~s}^{-1}$.

\subsubsection{Effects on the Rest of the Detector}

While the dimensions of the inner detector elements have not been changed, the presence of the stronger field in the inner detector region necessarily has significant effects. We discuss a few of them.

Effects on the Physics Capabilities of the Inner Detector The presence of a stronger field will have a beneficial effect on the capabilities of the inner tracking system. As noted above, the anticipated momentum resolution of the inner tracker for the 0.8 Tesla field in the $\mathrm{L}^{*} / \mathrm{GEM}$ design is $\Delta p / p \simeq 130 \% p(\mathrm{TeV} / \mathrm{c})$. In the high field case, if a similar tracking precision obtains, the momentum resolution in the inner tracking system will improve by a factor of six to $\Delta p / p \simeq 22 \% p(\mathrm{TeV} / \mathrm{c})$. With such resolution, reliable $(>5 \sigma)$ measurements of charge can be made up to momenta of $900 \mathrm{GeV}$, permitting, for example, the use of electron decay channels in the study of the production of high- $p_{t} W^{+} W^{+}$and $W^{-} W^{-}$pairs, the so-called guaranteed discovery reaction at the SSC [4]. Here reliable charge determination is 
needed to distinguish like-sign W-pairs, where the signal dominates, from unlike-sign pairs, which are dominated by background processes. The ability to use the electron and muon decay modes provides a nominal factor of four improvement in event rate over the case where only muons can be used. The rates for these processes are marginal. The improved efficiency provided by the electron channel is crucial for accessing this important area of physics. This reaction is discussed below in Section 2.1.

A potential problem in a detector with a high magnetic field is the spreading of the charged particles in a hadron jet before they enter the calorimeter, thereby reducing the effectiveness of jet clustering algorithms and particle isolation cuts. We have studied this in some detail and have concluded that it does not present a significant deterioration on the physics performance of the detector. This is discussed below in Section 2.3.

Effects on the Performance of the Inner Detection Elements Our proposed system places a number of constraints on the elements of the inner detector. For example, the momentum resolution in the muon system is limited primarily by multiple scattering in the material of the hadron calorimeter. Thus, we prefer that the calorimeter's radiator be a relatively low- $Z$ material such as iron (stainless steel) or copper, rather than lead or uranium. The high field probably precludes the operation of vacuum photodiodes for reading out scintillation light. Thus, for the case of the $\mathrm{BaF}$ option for EM calorimetry, alternative readout systems, such as proportional-wire readout using TAME, would have to be employed (assuming that the systematic calibration effects inherent in this scheme are resolved). We have investigated the effects on resolution of cryogenic liquid calorimeters, caused by the curling up of secondary particles produced in the entrance window before they reach the sampling medium, and see only a small degradation between 0.8 and 5 Tesla (see the discussion in Section 5.3). For a tracking radius of $75 \mathrm{~cm}$, a $5 \mathrm{~T}$ field will cause an increase in the number of curling tracks. Moreover, the increased Lorentz angle results in longer drift times in gas drift devices such as straw tubes. The combination of these effects results in serious occupancy problems for straw-tube-type inner tracking elements. We discuss problems in the inner tracking system in Section 5.1, where we argue that, at least for operation at $10^{33} \mathrm{~cm}^{-2} \mathrm{~s}^{-1}$, silicon strip and gas microstrip detectors will function well in the 5 Tesla environment.

\section{$1.3 \quad$ Summary}

In the following sections we provide supporting evidence for the claims made in this introduction and compare the capabilities of our proposed system to those of the $\mathrm{L}^{*} /$ GEM configuration for a variety of physics processes.

To summarize, we list some attributes of our proposed high field system:

- An iron return yoke is provided, minimizing stray magnetic fields in the experimental area and at the earth's surface. 
- Excellent muon momentum resolution, particularly at high momentum (better than that of $\mathrm{L}^{*} / \mathrm{GEM}$ for muons with $p_{t}>300 \mathrm{GeV}$ ).

- Good muon coverage out to $|\eta| \simeq 3.5$.

- Significantly relaxed alignment requirements.

- Very robust muon detection:

$-\Delta p / p \simeq 4 \%$ without reliance on the inner detection system; -redundant measurement of muons behind the iron return yoke.

- Simple muon triggering (behind $\geq 25 \lambda$ of material).

- A high magnetic field for the inner tracker system 


\section{Physics Capabilities of a High Field Detector}

The excellent triggerability and $\eta$ coverage of the muon detection system, the greatly improved resolution of the inner tracker that is provided by the high magnetic field, and the robustness that will enable operation at high luminosities, are all crucial for a variety of SSC "discovery physics" processes. In this section we discuss the reactions $p p \rightarrow W_{L}{ }^{+} W_{L}{ }^{+} X$ and Higgs $\longrightarrow Z^{\circ} Z^{*} \longrightarrow \mu^{+} \mu^{-} \mu^{+} \mu^{-}$in the context of the proposed high field configuration. In addition, we discuss the effects of the high field on the measurments of jet energies and on the isolation of leptons.

\section{$2.1 p p \longrightarrow W_{L}^{+} W_{L}^{+} X$}

The reaction $p p \longrightarrow W_{L}{ }^{+} W_{L}{ }^{+} X$ is central to the study of electroweak symmetry breaking at the SSC. In models with dynamical symmetry breaking, the cross section for this reaction is enhanced. It has been shown on rather general grounds that if there is no Higgs particle with a mass that is accessible at the SSC, the interactions of longitudinally polarized W's (and Z's) must become strong for W-W cm energies above $1 \mathrm{TeV}$ [4]. (Indeed, this is the flip side of the SSC's "promise to elucidate the nature of electroweak symmetry breaking" [5].) In this event, the cross section for the reaction $p p \longrightarrow W_{L}^{+} W_{L}^{+} X \longrightarrow \mu^{+}\left(e^{+}\right) \mu^{+}\left(e^{+}\right)$has been estimated to be between 5-10 fb, corresponding to only 50-100 events per SSC year with no acceptance factors taken into account [6].
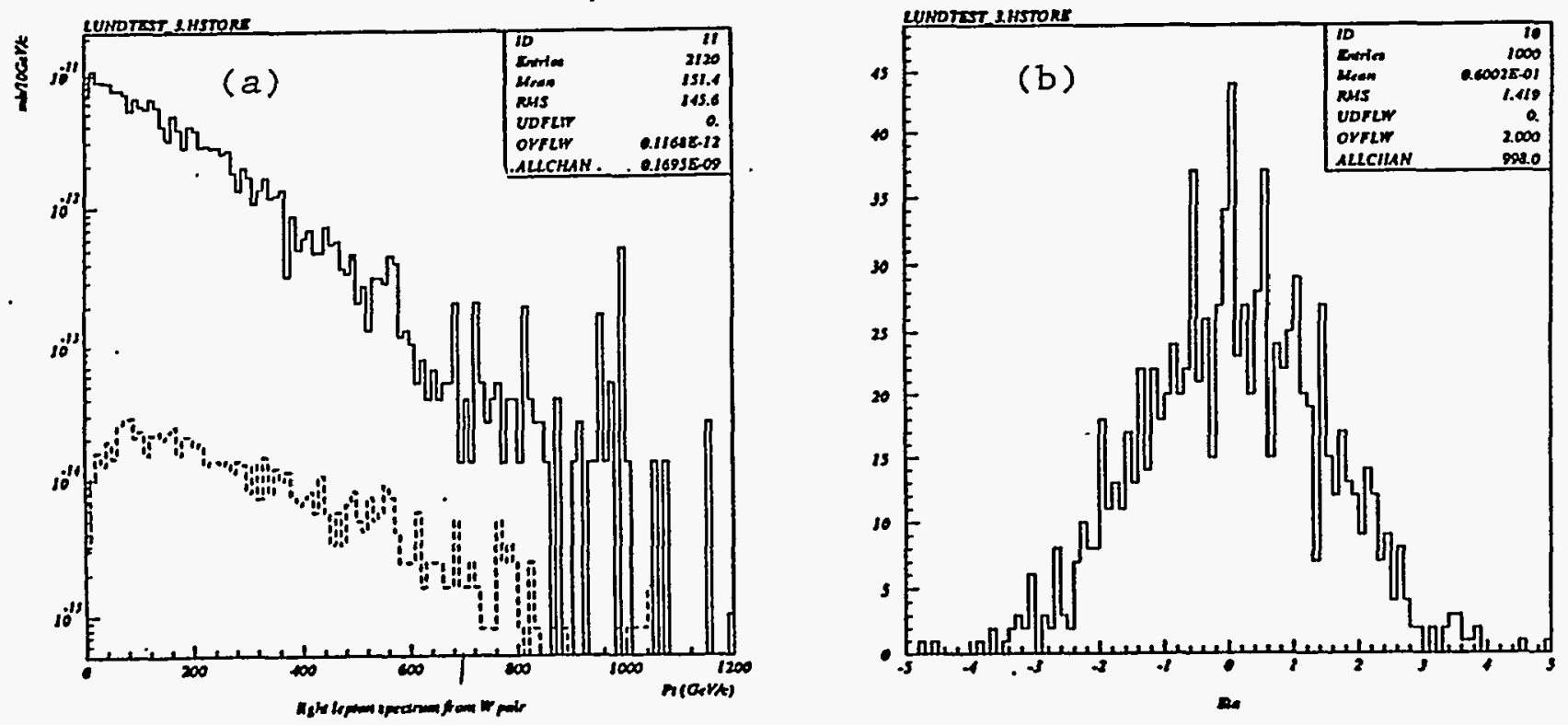

Figure 12: (a) Momentum and (b) $\eta$ distribution of like-sign leptons from the $p p \longrightarrow W_{L}{ }^{+} W_{L}^{+} X$ (dashed) interaction and the opposite-sign leptons from WW production (solid).

Figure 12 shows the momentum (a) and $\eta(b)$ distribution of like-sign leptons from 
the $p p \longrightarrow W_{L}{ }^{+} W_{L}{ }^{+} X$ interaction and the opposite-sign leptons from WW production. Backgrounds in the opposite-sign lepton pair channels from $q \bar{q} \longrightarrow W^{+} W^{-}$are of order $10^{2} \sim$ $10^{3}$ times higher than the signal, and preclude the use of the unlike-sign channel for these studies. The dominant backgrounds in like-sign cannel are from $t \bar{t}$ and $t \bar{t} j e t$ events [7], which is even more severe in the case of opposite-sign leptons. In order to avoid contamination of the like-sign signal from misidentified unlike-sign backgrounds, one needs excellent sign determination. To preserve a measureable rate, this capability is needed for both electrons and muons with energies up to $\sim 1 \mathrm{TeV}$ and for $|\eta|$ values as large as 3.0.

The high field in the inner tracking region extends the momentum range for electron sign determination by a factor of six over the $\mathrm{L}^{*} / \mathrm{GEM}$ design ${ }^{1}$ (from $\sim 150$ to $\sim 1000 \mathrm{GeV} / \mathrm{c}$ ). In addition, our proposed geometry covers a larger $|\eta|$ region for muons-the $\mathrm{L}^{*} / \mathrm{GEM}$ design has lower acceptance for two muons from $p p \longrightarrow W_{L}+W_{L}+X$ than our high field proposal. The combined effect of the additional electron momentum and muon $|\eta|$ coverage results in a difference of about a factor of five in the efficiency for these events. Thus, our proposed high field configuration addresses a serious deficiency in ability of an $\mathrm{L}^{*} / \mathrm{GEM}$ style detector, and enables the investigation of this crucial physics topic.

\section{$2.2 \quad H i g g s \longrightarrow Z^{\circ} Z^{*} \longrightarrow \mu^{+} \mu^{-} \mu^{+} \mu^{-}$}

This reaction has been suggested as a method for seeing the Higgs if its mass is between about 140 and $180 \mathrm{GeV} / \mathrm{c}^{2}$. Here the natural width of the Higgs is expected to be narrow, less than $1 \mathrm{GeV} / \mathrm{c}^{2}$, and there is a premium on good momentum resolution for $p_{t} \sim 100 \mathrm{GeV} / \mathrm{c}$ muons. While the $p_{t}$ resolution for the high field muon system is superior to that of the $\mathrm{L}^{*} / \mathrm{GEM}$ approach for $p_{t}>350 \mathrm{GeV} / \mathrm{c}$, the $\mathrm{L}^{*} / \mathrm{GEM}$ muon system is superior for lower momentum muons. Thus, this reaction has been proposed as one for which the high field detector being proposed here is clearly inferior to $\mathrm{L}^{*} / \mathrm{GEM}$.

However, the high field configuration proposed here has some advantages over $\mathrm{L}^{*} / \mathrm{GEM}$ : the muon coverage extends to larger values of $|\eta|$, and the higher field results in a superior $p_{t}$ resolution for the inner tracking system. Figure 13 shows the maximum $|\eta|$ value of the four final-state muons from an ISAJET simulation for a Higgs mass of $140 \mathrm{GeV} / \mathrm{c}^{2}$. (Here we restrict ourselves to final states where all four muons have $p_{t} \geq 5 \mathrm{GeV} / \mathrm{c}$.) From the figure it is apparent that expanding the $|\eta|$ coverage from 2.5 to 3.5 results in an approximate $40 \%$ increase in acceptance. The extra $|\eta|$ coverage for muons also increases the acceptance for the Higgs $\longrightarrow Z^{\circ} Z^{*} \longrightarrow \mu^{+} \mu^{-} e^{+} e^{-}$, which is twice as frequent. Figure 14 a shows a comparison of the high-field and $\mathrm{L}^{*} / \mathrm{GEM} p_{t}$ resolutions for $p_{t}=25 \mathrm{GeV} / \mathrm{c}$ muons in the external muon systems. The superiority of the $\mathrm{L}^{*} / \mathrm{GEM}$ external muon system for $p_{t} \sim 25 \mathrm{GeV} / \mathrm{c}$ muons is apparent. However, Fig. 14b shows a comparison when the inner tracking system (covering the region $|\eta| \leq 2.5)$ is included. Here, the high field in the inner tracking region results in a significantly better $p_{t}$ resolution at $p_{t} \sim 25 \mathrm{GeV} / \mathrm{c}$.

\footnotetext{
${ }^{1}$ We consider a $\sim 5 \sigma$ charge determination to be necessary for eliminating a background that is $10^{3}$ times the signal.
} 


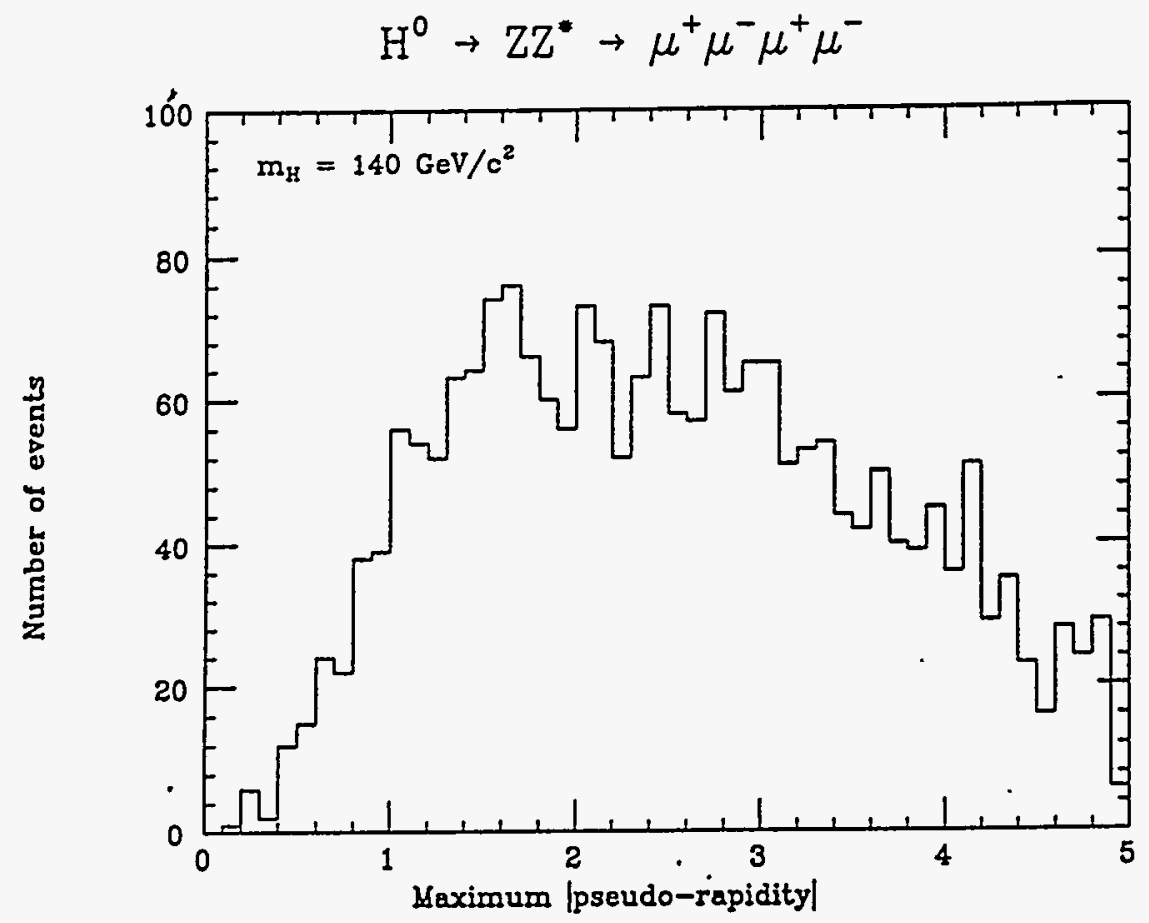

Figure 13: The maximum $|\eta|$ value for muons from the reaction Higgs $\longrightarrow Z^{\circ} Z^{*} \longrightarrow \mu^{+} \mu^{-} \mu^{+} \mu^{-}$.
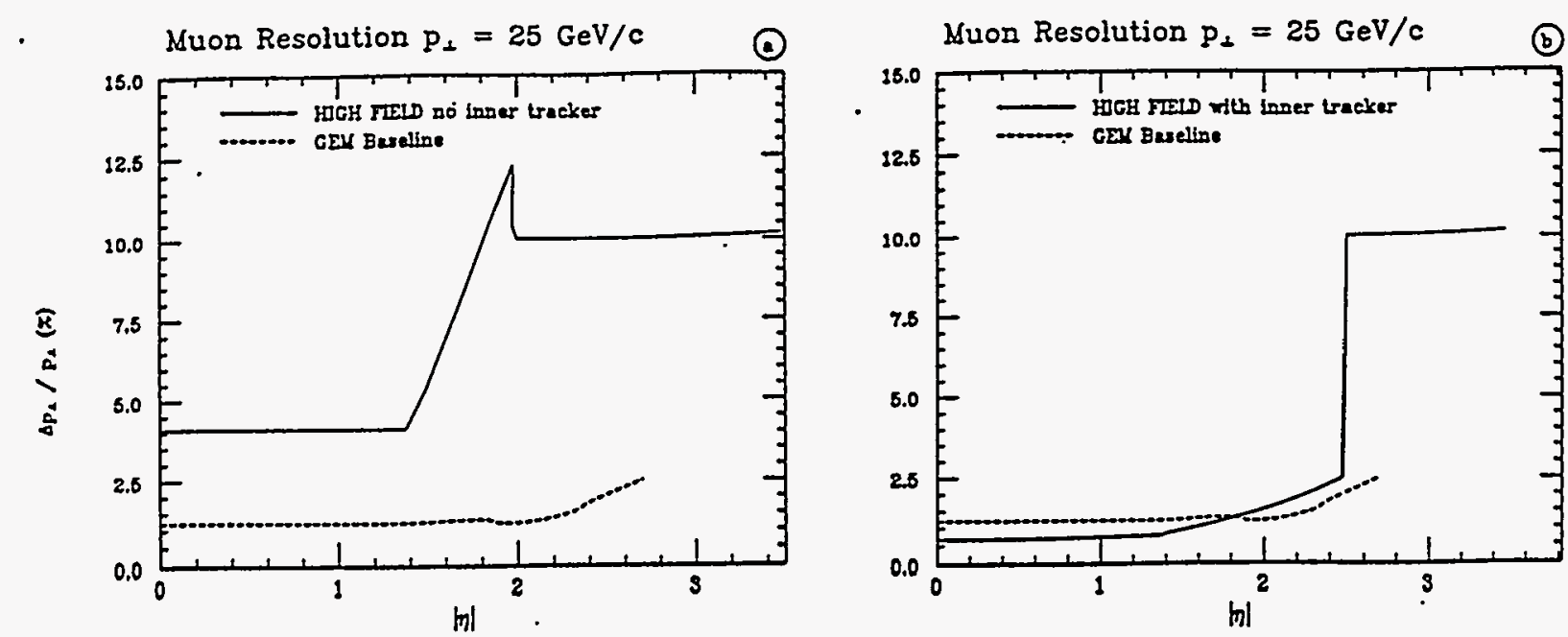

Figure 14: $\Delta p_{t} / p_{t}$ for the high field (solid line) and baseline (dashed line) detectors for $p_{t}=25 \mathrm{GeV} / \mathrm{c}$ muons. (a) External muon detectors only. (b) Including the inner tracking system. 

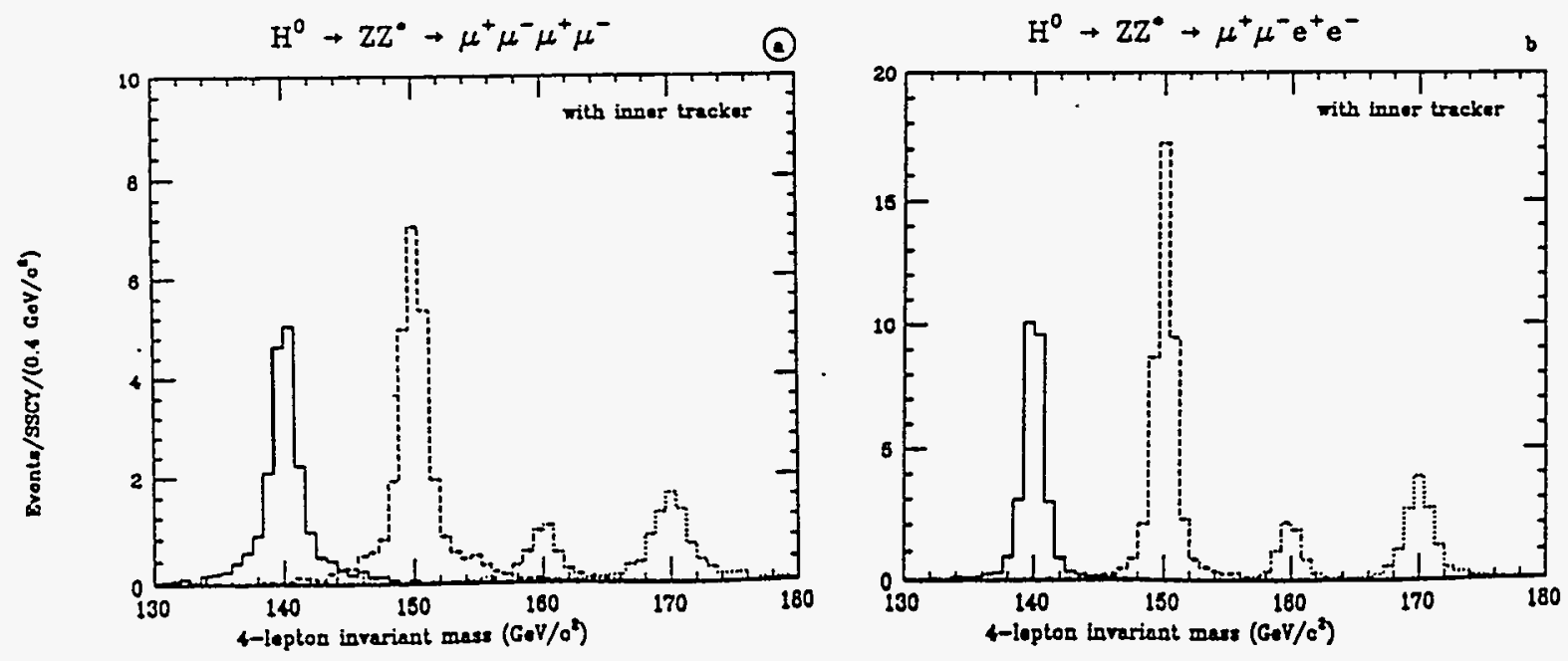

Figure 15: Higgs mass spectra for the Higgs $\longrightarrow Z^{\circ} Z^{*}$ for the case of: (a) $\mu^{+} \mu^{-} \mu^{+} \mu^{-}$and (b) $\mu^{+} \mu^{-} e^{+} e^{-}$final states.

Figure $15 \mathrm{a}$ is a composite distribution showing the results of an idealized study (no backgrounds are included) for Higgs masses of $140,150,160$, and $160 \mathrm{GeV} / \mathrm{c}^{2}$, where the inner tracking information is used for muons with $|\eta|<2.5$. All muons are required to have $p_{t}>5 \mathrm{GeV} / \mathrm{c}$. The results are normalized to an "SSC year" (an integrated luminosity of $10^{40} \mathrm{~cm}^{-2}$ ). The narrow peak corresponds to events where all muons are contained in the inner tracker; the broad tail results from those events where one or more muons are measured in the forward toroids. The narrow peak corresponds to a mass resolution of $0.8 \mathrm{GeV} / \mathrm{c}^{2}$, which is substantially better than the corresponding mass resolution for the $L^{*} / G E M$ design (as shown in Fig. 2.2 of the GEM EOI). Figure 15b shows the corresponding results for the Higgs $\longrightarrow \mu^{+} \mu^{-} e^{+} e^{-}$mode, where we assume that the electrons are detected in an EM calorinmeter with a resolution of $7.5 \% / \sqrt{E(\mathrm{GeV}}$ combined in quadrature with a constant term of $0.5 \%$. For the high field case, the momentum resolution of the inner tracker is superior to the energy resolution of the EM calorimeter for momenta below about $50 \mathrm{GeV} / \mathrm{c}$. Thus, since roughly half of the final-state leptons from this channel are below $50 \mathrm{GeV} / \mathrm{c}$, the mass resolutions for the two cases are quite similar. Comparing the results in Figs. 15a and $\mathrm{b}$ with those of Fig. 2.2 in the GEM group's EOI indicates that the high field detector has a significant advantage over the $L^{*} / G E M$ design for this reaction when information from the inner tracker is employed.

\subsection{The Effect of the Field on Jet Detection}

The detection of hadron jets at the SSC will rely primarily on calorimetry. A potential harmful effect of the high magnetic field is the "spreading" of particles in the jets with potential deterioration of jet energy and angular resolution. We have studied this effect for 


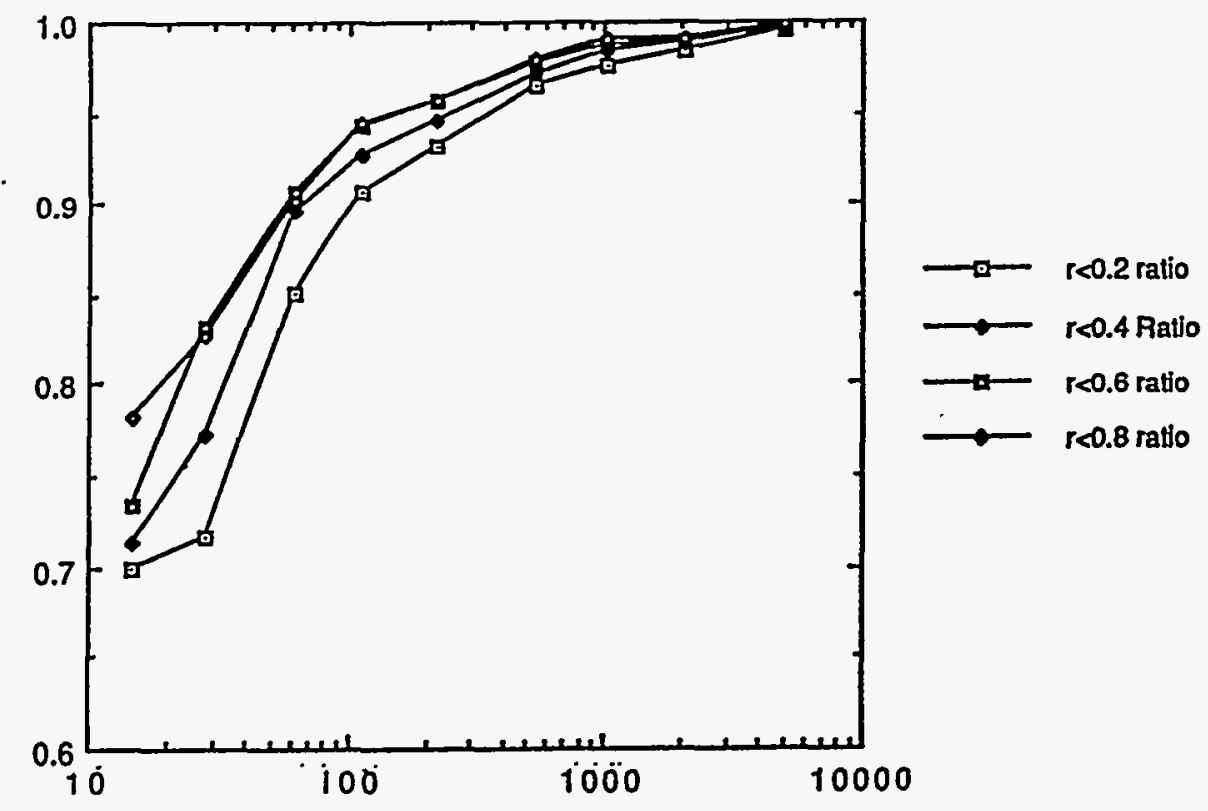

Pi Jet (GoV/c)

Figure 16: The ratio of the jet energy contained in a cone $\mathrm{R}$ for $B=5 \mathrm{~T}$ to that contained in the same size cone for $B=0$ vs the jet energy for various $\mathrm{R}$ values.

jets with momenta ranging from 20 to $5000 \mathrm{GeV}$. The relevant parameters are the fraction of the jet's "potentially observable energy," i.e., the energy of the parent parton minus the energy of neutrinos, that is contained with an $R$ cone of some value, and the rms fluctuations of this fraction. We define $R$ in the standard fashion as

$$
R=\sqrt{\phi^{2}+\eta^{2}}
$$

The relevant issue here is the size of fluctuations since even with no magnetic field one does not generally detect all the jet energy and an $R$-and energy-dependent correction factor must be applied. Figure 16 shows the ratio of the energy fraction contained within various $R$ cones in a $5 \mathrm{~T}$ field to that with no magnetic field. Figure 17 shows the energy fractions and the rms fluctuations of these fractions for an $R$ value of 0.8 . The conclusion is that for low energy jets, a high field reduces the energy in an $R$ cone relative to the no-field case. However, the rms fluctuations are very nearly the same in both cases. Thus, although the higher field results in a larger correction factor, the jet resolution is not seriously compromised.

\subsection{The Effect of the Field on Isolation Cuts}

Another concern is the effect of the field on isolation cuts of leptons outside the jet. There is the potential for the field to "sweep" particles from the jet nearer the otherwise isolated lepton and destroy the efficacy of the isolation cut. As a stringent test we study the decay

$$
p p \longrightarrow t \bar{t}+X ; \quad t \bar{t} \longrightarrow W^{+} b \quad W^{-} \bar{b} \longrightarrow \mu^{+} \mu^{-} j e t s
$$


$E(<0.8) / E($ let $)$

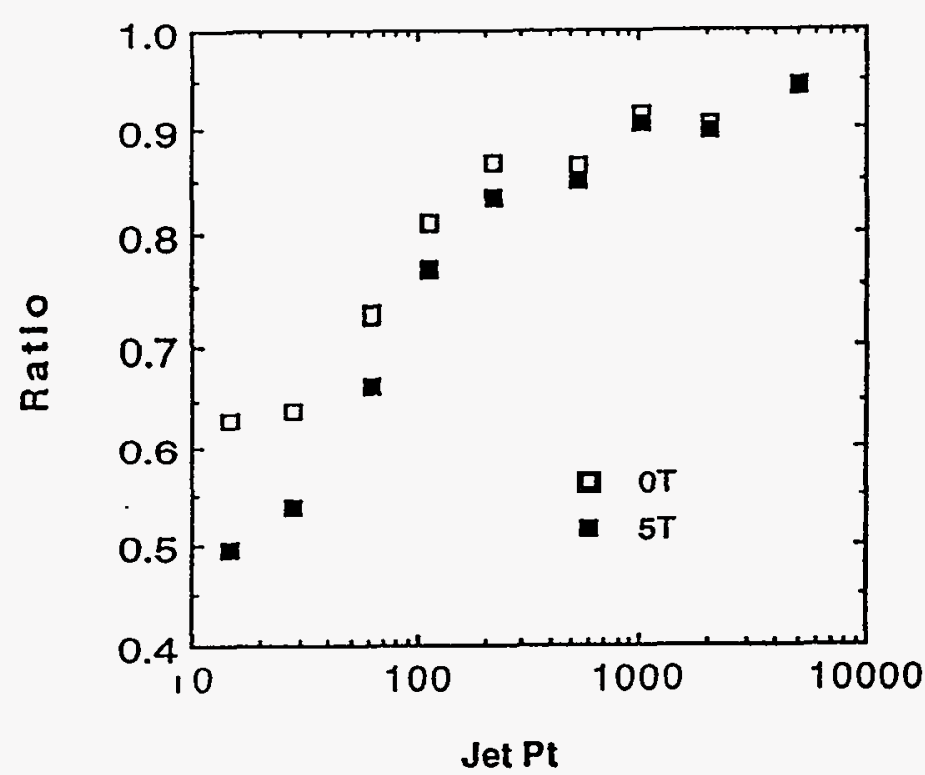

RMS [ $E(<0.8) / E($ jet $)]$

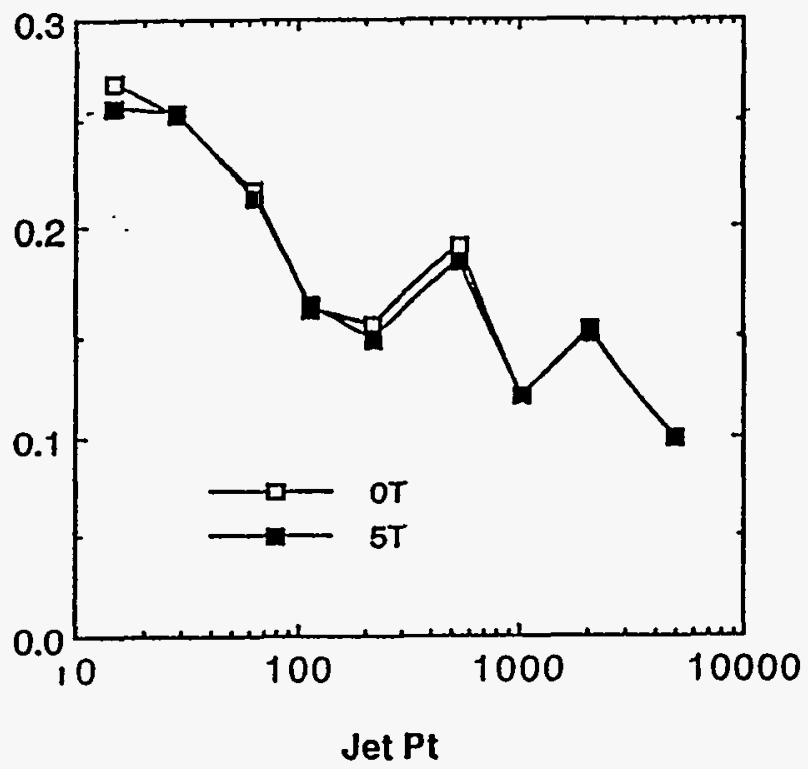

Figure 17: The jet energy fractions and their rms fluctuations for an $R$ value of 0.8 , for $B=0$ and $B=5 \mathrm{~T}$.

for a t-quark mass of $200 \mathrm{GeV} / \mathrm{c}^{2}$. The dominant backgrounds to this signal are the muons from the decays of $b$ and $c$ quarks. In Fig. 18 we plot the muon $p_{t}$ spectra for the $t \bar{t}$ signal events (dotted line), the background from $b$ and $c$ decays prior to any cuts (solid line), and the $b$ and $c$ background after the application of the isolation cut requiring the total transverse energy in an $R=0.4$ cone around the muon be below $10 \mathrm{GeV}$ (dashed line). (This is the cut used in many simulated top analyses for SSC experiments.)

Figure 18 shows the effect for no magnetic field (a) and effect for a $5 \mathrm{~T}$ field (b). The figure of merit is where the signal level intersects the background, around $40 \mathrm{GeV}$ in both cases. We conclude that the $5 \mathrm{~T}$ field has no significant effect on isolation cuts aimed at observing the isolated leptons.

\section{Muon Detection Simulation}

In this section we consider the details of the design of the post-coil muon tracking system. An important consideration in the design of a muon spectrometer for SSC detectors is the effect of showers induced by the muon traversing material (muon radiation and delta-ray production). These accompanying showers may obscure the muon track and worsen the effective resolution of the system, and have the potential for producing fake high $p_{t}$ triggers. In order to optimize the cell size and the number of layers required for accurate and reliable measurements, the nature of these backgrounds must be well understood. We are studying these effects in our proposed geometry by means of a GEANT simulation.

As described before, there are three separate muon track measurements, the "inner 

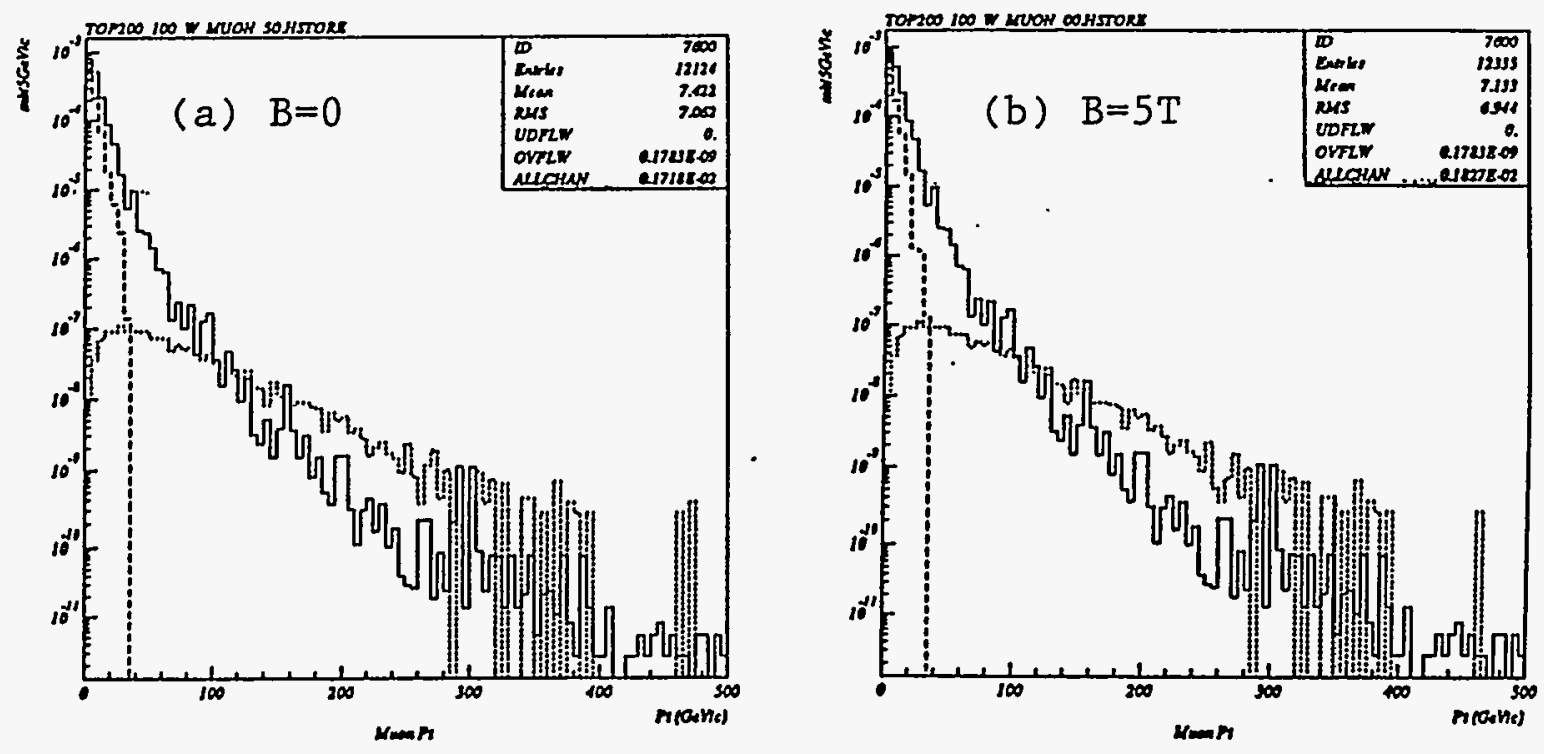

Figure 18: The transverse momentum distribution of the isolated lepton from a $200 \mathrm{GeV}$ top decay (dotted line), $c$ and $b$ quark decays (solid line) and $c$ and $b$ quark decay after isolation cut (dashed line) for (a) no magnetic field, (b) a $5 \mathrm{~T}$ field.

tracker" surrounding the interaction point, the "middle tracker" in the region between the coil and the iron flux return, and the "outer tracker" beyond the iron yoke (see Fig. 4). Here we summarize initial results from a study of the effects of muon-induced showers on the ability to measure the angle $\alpha$ in the middle tracker. When a particle accompanying the muon passes through a drift chamber element closer to the wire than the muon does, it causes an incorrect position measurement and, therefore, a worsening of the resolution. (Not yet considered in this program is the effect on pattern recognition and triggering of having several cells in a layer affected in this manner. While this has to be investigated in detail, we expect that our specific proposed geometry, with its three separate muon-track measurements, has an advantage over that of $\mathrm{L}^{*} / \mathrm{GEM}$ design, both in resolving track ambiguities and in rejecting the erroneous triggers introduced by such particles.)

As discussed in Section 1.2, the uncertainty in the measurement of the outgoing angle $\alpha$ has a contribution from multiple scattering in the calorimetry (here we assume a thickness of $\left.110 x_{r l}\right)$ and in the coil $\left(20 x_{r l}\right)$ of $\sigma \simeq 0.11 \mathrm{mrad} / p(\mathrm{TeV} / \mathrm{c})$, provided that the exit position is well measured. Thus, for a measurement to be limited by this multiple scattering uncertainty, the muon system must not provide any significant additional contribution to the determination of $\alpha$. For a $1 \mathrm{TeV} / \mathrm{c}$ muon, and a $\sim 1 \mathrm{~m}$ tracking length, this amounts to a spatial position resolution at the track ends of $\leq 50 \mu \mathrm{m}$. Assuming a typical single cell resolution is near $100 \mu \mathrm{m}$, a minimum of 4 layers of cells at the exit of the coil and 4 layers before the flux return will be required to preserve the limiting value of resolution. However, because of muon-induced showers, hit measurements can be compromised by the presence of any accompanying particles. These extraneous hits can be recognized by the track fitting algorithm and removed from the fit, but, unfortunately, at the cost of reducing the number 


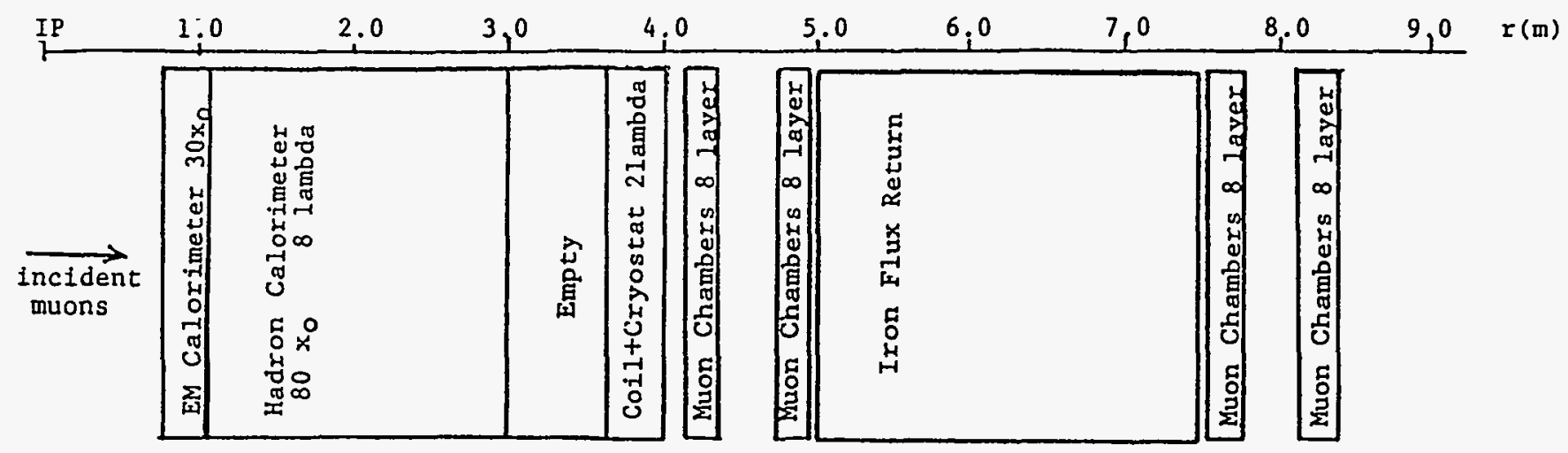

Figure 19: The muon chambers layer geometry used in the simulation.

of cell layers in the fit and a worsening of the resolution. The frequency of occurrence of these compromised cells and any impact on the measurement quality can be reduced by decreasing the cell size, or increasing the redundancy of the system by adding more layers of cells. Ultimately one has to optimize both the cell size and numbers of layers.

\subsection{The Simulation}

To understand backgrounds from muon-induced showers, it is very important to simulate in detail the layers of material before the muon system as well as the muon system itself. For this study the simulated calorimeter was a homogeneous mixture of absorber $(\mathrm{Pb}$ in the electromagnetic portion and $\mathrm{Fe}$ in the hadronic section) and scintillator. The details of the coil and chambers used for the simulation are shown in Fig. 19. For the muon chambers, the cell design proposed by L. Osborne et al. [8] is used. The cells have $2 \mathrm{~mm}$ thick $\mathrm{Al}$ walls (it was recently proposed to reduce this), and $2.5 \mathrm{~cm}$ square drift cells with a sense wire down the center. The resolution of these cells has been demonstrated to be $\sigma \sim 80 \mu \mathrm{m}$ [9]. We use the GEANT program in this study; a GEANT simulation of showers induced by $0.5 \mathrm{TeV} / \mathrm{c}$ muons was found to be consistent with measurements made in a Fermilab muon beam. Because charged particles with energy below $1 \mathrm{MeV}$ would not penetrate the $2 \mathrm{~mm}$ thick Al walls of the drift chambers, the energy cutoffs in GEANT for electrons and photons in the material of the calorimeter, coil and flux return were set to $1 \mathrm{MeV}$. However, in the material of the drift chamber walls and in the Ar-isobutane cell gas, $10 \mathrm{KeV}$ cutoffs were used in order to simulate the sensitivity of the drift chamber cells to very low energy charged particles.

A configuration of two superlayers (SL), with each superlayer consisting of 8 layers of cells, was positioned in the middle tracking region as shown in Fig. 19. By adjusting the thickness of the flux return iron, the field strength in the middle-tracking region can be "tuned" from zero to a few kG. To consider the benefit of a field in this region, the simulation was performed both at zero field and $2 \mathrm{kG}$, with the field direction along the length of the cells. Single muons with incident momenta of $1 \mathrm{TeV} / \mathrm{c}$ are propagated through the detector. 
Thus far, only a $90^{\circ}$ incidence angle has been simulated. The position and 4-momentum is recorded for particles in the chamber layers as they cross the plane containing the wires (mid-point of the cell). After the GEANT simulation, the event records are then read back by an analysis routine which constructs the response of individual cells. The cells in evennumbered layers are offset by $1 / 2$ cell width from those in the odd-numbered layers. The positioning of the entry point of the muon relative to the "wire" in the first layer of the first superlayer can be adjusted. As a test, the entire configuration of cells was shifted with respect to the muon entry point by $0.62 \mathrm{~cm}$ in the $\mathrm{X}$ direction, with no discernable change observed in the results.

\subsection{Results}

Figure 20 shows, both for a $B=0$ (a) and $B=2 \mathrm{kG}$ (b) field in the region of the middle tracker, the distribution of the total number of cells that are hit by the $1 \mathrm{TeV} / \mathrm{c}$ incident muon and its accompanying particles. As expected, this distribution peaks at 16 (total number of layers in the middle tracker), and has a tail for larger number of hits coming from muon-induced showers. As noted above, this study only concerns itself with the 16 cells that the muons traverse. Figure 21 shows the number of total layers (out of 8 ) in each superlayer in which the muon was nearest to the wire ("good hit") in the traversed cell. The probability that all 8 layers in a superlayer are "good" ranges from $67 \%-69 \%$ for zero field, to $70 \%$ $76 \%$ for $2 \mathrm{kG}$ field. As expected, the presence of some magnetic field in the tracking region serves to suppress the background from muon-induced delta-rays by sweeping them away. Also, as expected, the response in the superlayer further away from the material of the coil (SL2) is somewhat better than in the closer one (SL1).

As noted above, the required spatial resolution can be obtained using a minimum of four layers in each superlayer. To consider this requirement in detail, we examine the probability of having at least four "good" hits in the first $N$ layers of a superlayer, for $N=4,5,6,7$, and 8 . This is plotted in Fig. 22, both for SL1 and SL2, and for $B=2 \mathrm{kG}$. The probability for at least four "good" cells in SL2 increases from $80 \%$ ( $86 \%$ at $2 \mathrm{kG}$ ) for N=4 layers to $94 \%$ ( $96 \%$ at $2 \mathrm{kG}$ ) for $\mathrm{N}=8$ layers. Finally, it was found that for a middle tracker with eight layers of chambers in each of two superlayers, $92.5 \%(94.9 \%$ at $2 \mathrm{kG})$ of $1 \mathrm{TeV} / \mathrm{c}$ muons provide at least four "good" hits in each superlayer.

\subsection{Conclusions}

Of order 16 layers, of $2.5 \mathrm{~cm}$ wide cells, will provide sufficient redundancy for efficient muon measurements in the middle tracker. With multi-hit electronics, the number of compromised muon cells could be reduced, and thereby provide the possibility of reducing the number of layers, and the corresponding channel count and costs. Further simulation will consider other candidate technologies for muon detection and problems in pattern recognition in high multiplicity events, where the linkage between hits in the outer tracking region to those in 

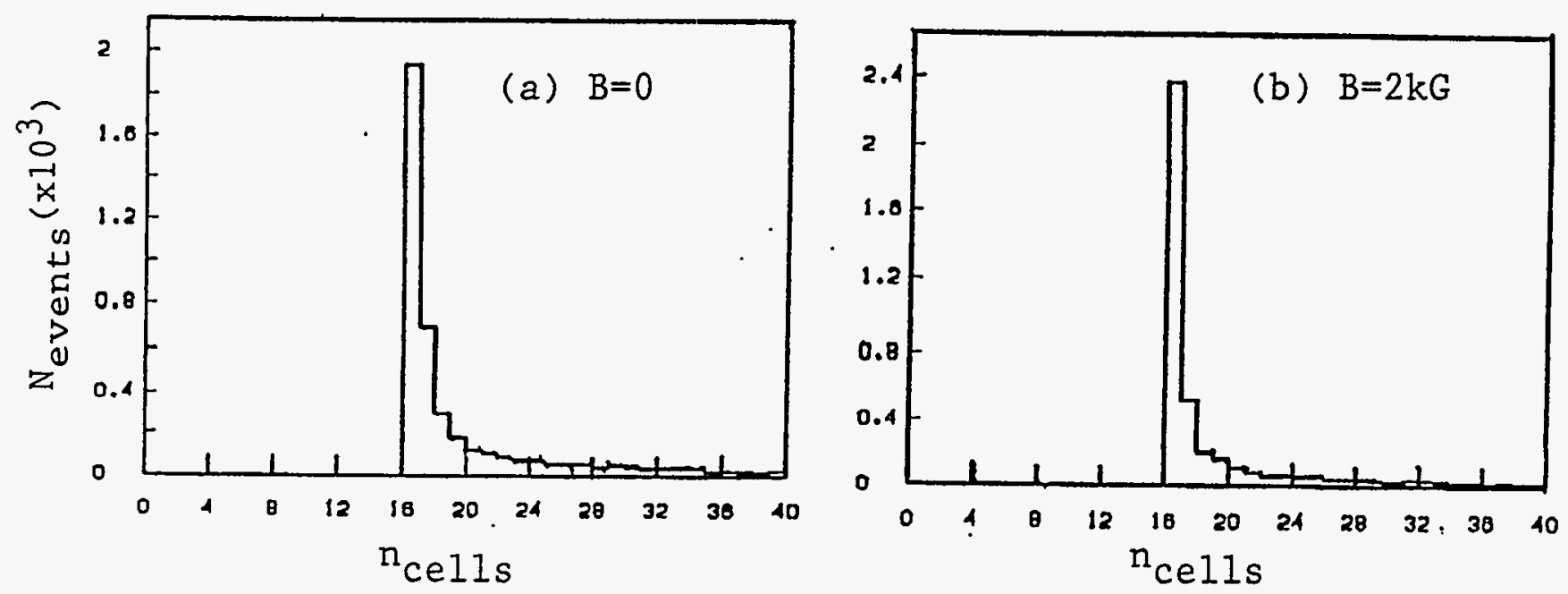

Figure 20: The distribution of the total number of cells in the middle tracker which are hit by the $1 \mathrm{TeV} / \mathrm{c}$ incident muon and its accompanying particles.
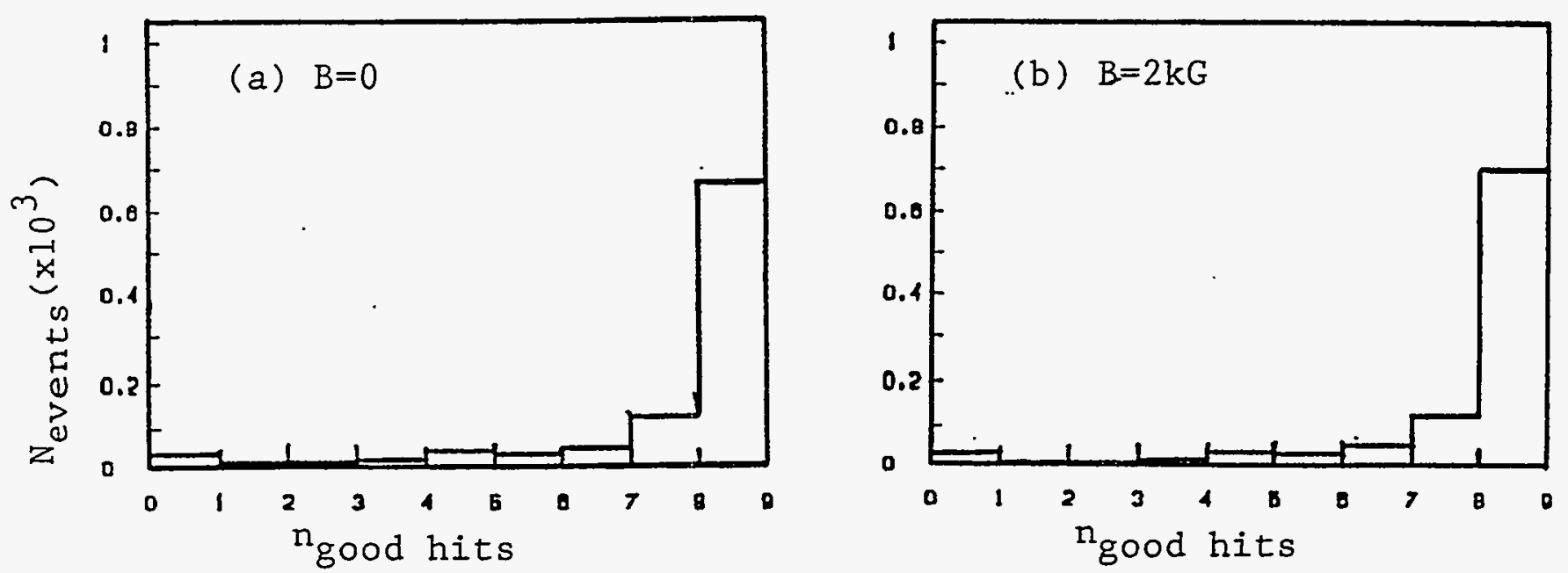

Figure 21: The number of total layers per superlayer (out of 8) in which the muon came nearest to the wire ("good hit") in the traversed cell. Comparison is shown for the two superlayers SL1 and SL2 for $B=2 \mathrm{kG}$ 

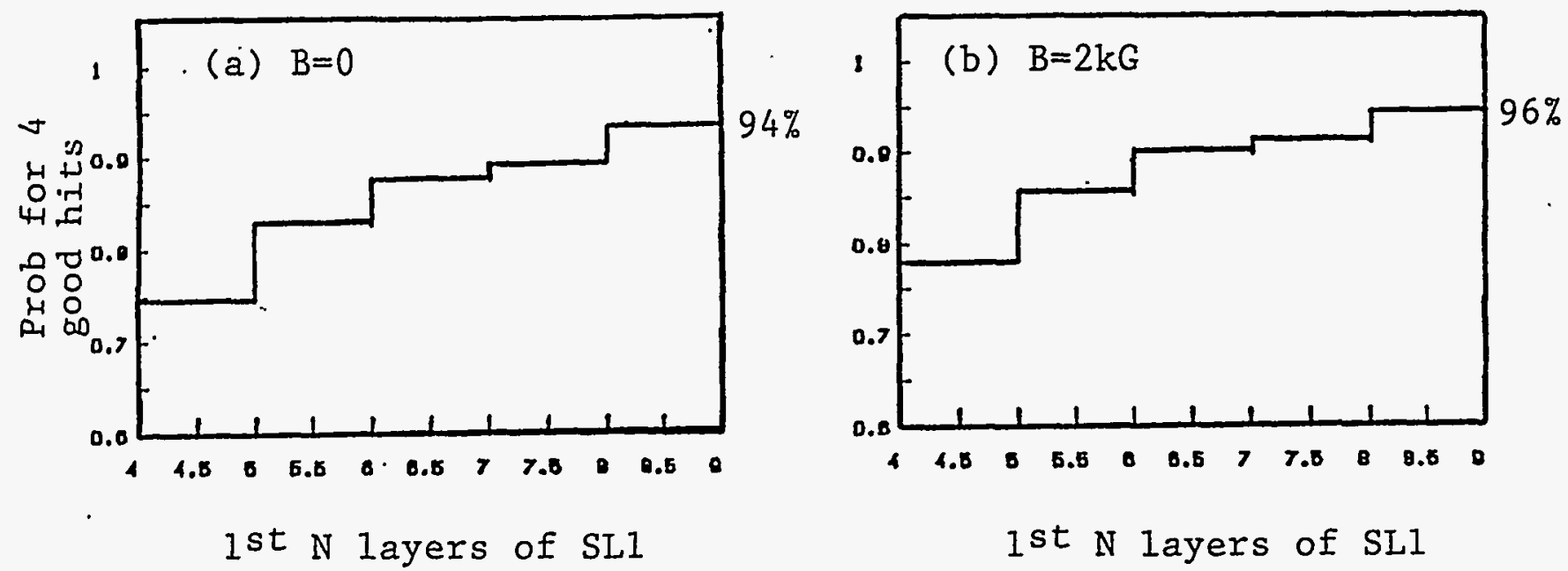

Figure 22: The probability of having at least four good hits in the first $\mathrm{N}$ layers of the superlayer just outside the coil, for $N=4,5,6,7$, and 8 .

the middle tracker will be used to help identify muons.

\section{Magnet and Mechanical Support}

Our proposed geometry offers significant simplification in the mechanical support system and the coil. The iron flux return serves as an excellent structural element for supporting the muon tracking systems. Coupled with the less stringent alignment requirements for these systems, this offers potential cost and scheduling advantages. In addition, the coil is sufficiently small to allow for transportation to the laboratory site, thus de-coupling the magnet production schedule from land acquisition, factory construction, and other activities. We are undertaking two studies on the mechanical support and the magnet coil, and report preliminary but encouraging results.

\subsection{Magnet}

To keep the dimensions of the inner tracker and calorimetry the same as the current $L^{*} / G E M$ design, the superconducting magnet has an inner radius of $3.5 \mathrm{~m}$ and a total length of $15 \mathrm{~m}$ so as to enhance the $\eta$ acceptance of the central muon system.

A preliminary examination by KEK magnet experts concluded that the magnet was "feasible from the point of view of magnet technology." The main paramters of the solenoid are listed in the following table. 


\begin{tabular}{|l|l|}
\hline \hline \multicolumn{2}{|c|}{ Magnet Parameters } \\
\hline Central field & 5 Tesla \\
Maximum field in the coil & 5.5 Tesla \\
Current density in the coil & $23 \mathrm{Amp} / \mathrm{mm}^{2}$ \\
Stored energy & $5.58 \mathrm{GJ}$ \\
Axial compression (coil midplane) & 31,730 tons \\
Radial expansion pressure & $107 \mathrm{~atm}$ \\
Maximum hoop stress & $22 \mathrm{~kg} / \mathrm{mm}^{2}$ \\
\hline \hline
\end{tabular}

The estimated maximum hoop stress of $22 \mathrm{~kg} / \mathrm{mm}^{2}$, which is three times that in the AMY coil, could be accomodated using a hard-copper conductor enclosed in a stainless-steel sheath (the AMY conductor uses hard copper only). The development of this conductor will require some $R \& D$. The large axial compression forces can be reduced by separating the coil into a few longtitudinal segments. Cooling methods, the cross section of the conductor, the detailed structure of the coil, quench protection systems, etc., are currently under study.

Iron is provided for the flux return, bringing the fringing field at $60 \mathrm{~m}$ from beam to less than 4 gauss. The current structural design calls for two separate half-coils, supported from the iron by a diaphragm as shown in Fig. 23. The diaphragm also supports a stainless steel cylinder within which are mounted the calorimeter and inner tracker. The "barrel" iron, as shown in Fig. 24, can be built in either eight or sixteen sectors, with eight preferred for reasons of economy (fewer welds, supports, bolts, etc.).

Figure 25 shows a field map for the configuration discussed above. Figure 26 shows how the magnetic field drops off with $\mathrm{Z}$, the vertical distance from the beam line. The cost of the coil has been estimated as between $\$ 70-100 \mathrm{M}$ [10]. We are currently proceeding with a more thorough estimate of both the cost and schedule of magnet construction. The cost of the coil could be reduced with little compromise in the performance for muons if ordinary ferromagnetic iron is used as the radiator in the hadronic calorimeter. This boosts the magnetic field in that region at no cost in ampere-turns in the coil. In this case, the calorimeter support system has to accommodate magnetic forces as well as gravitational forces, adding to its expense. An optimization, based on detailed engineering designs, has to be done.

\subsection{Mechanical Assembly and Support}

The inner detector is assembled into a cylinder that captures the superconducting coil and is supported from its ends, as well as by a mid-plane diaphragm (Fig. 23). While the study of mechanical design is in a preliminary stage, one can draw some conclusions above the advantages of our proposed geometry over that of the $L^{*} / G E M$ configuration:

1. The schedule for the construction and implementation of the coil is relatively independent of the development of the rest of the detector. 


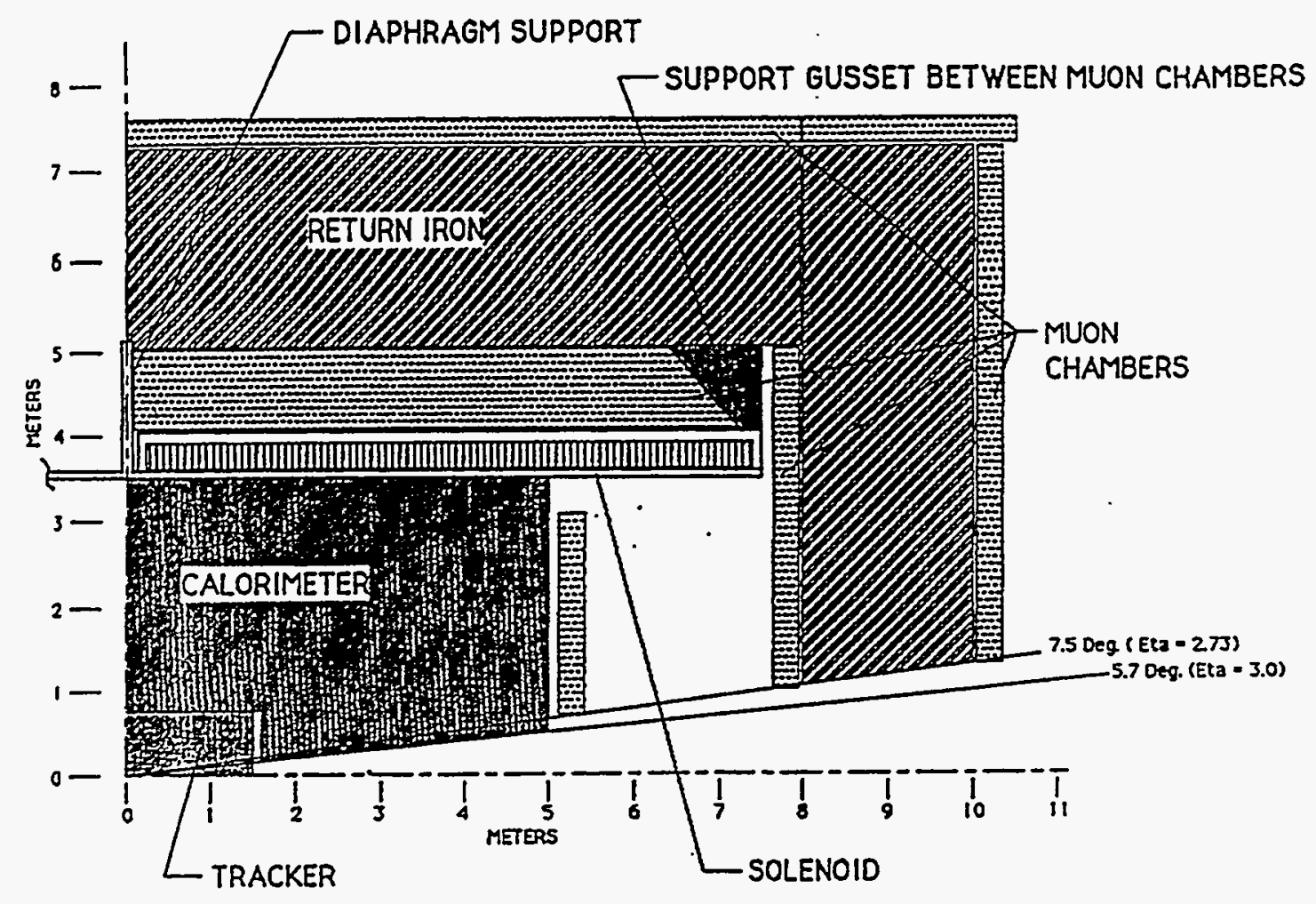

Figure 23: A view of the high field coil showing the diaphragm support in the middle of the coil.

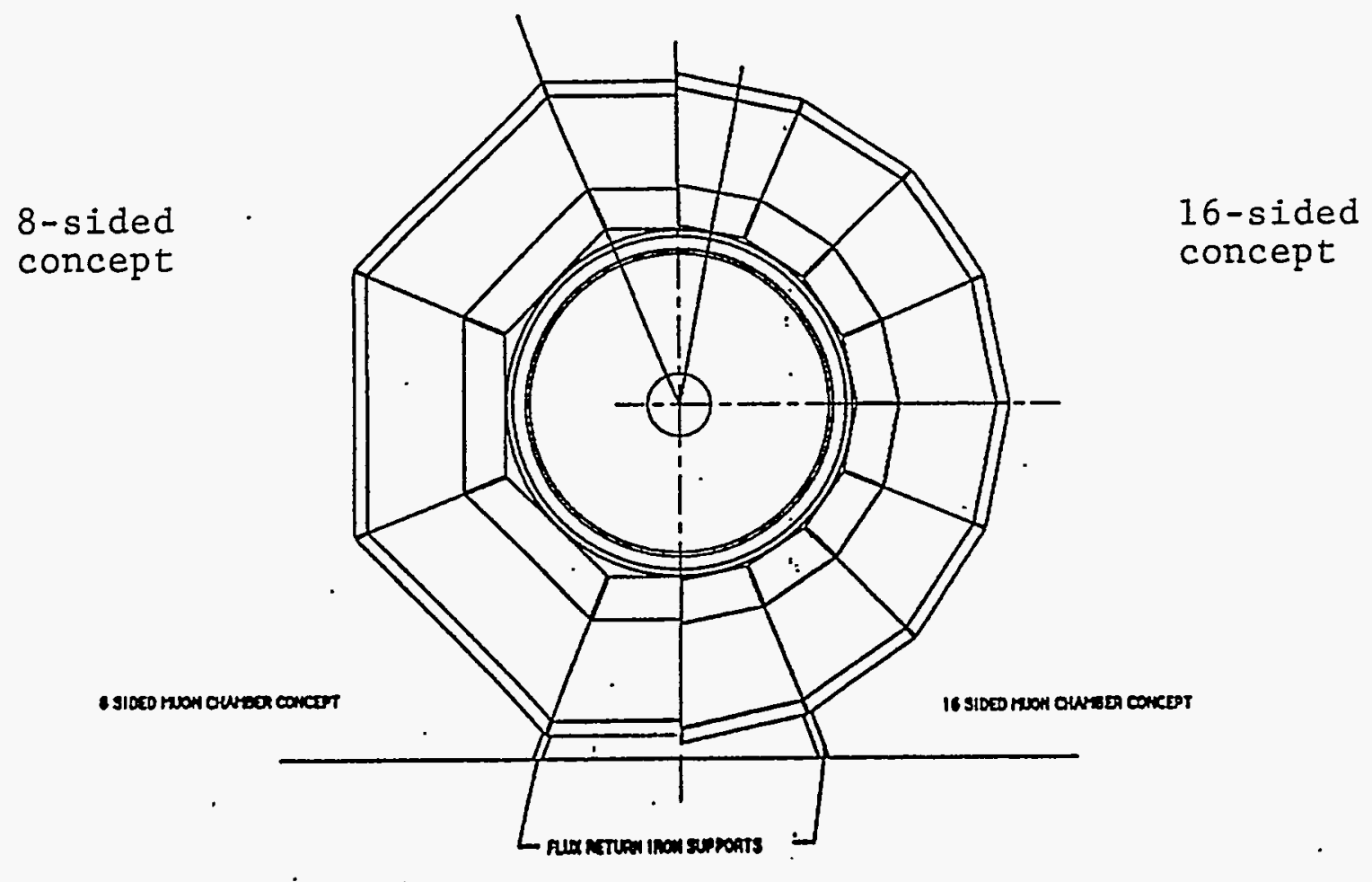

Figure 24: An end view of the barrel iron showing the overall support. 


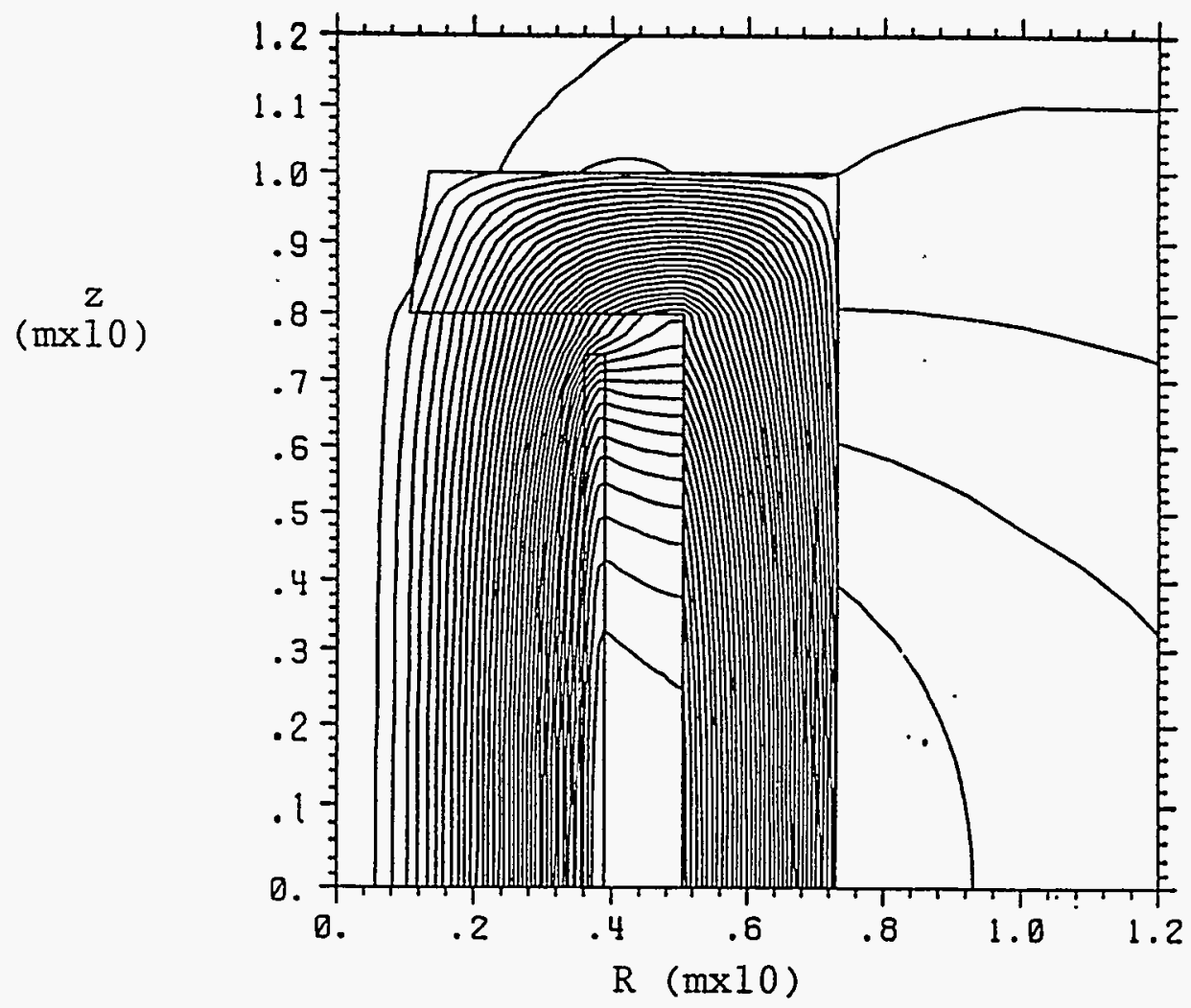

Figure 25: A field map showing the field lines for the High Field coil.

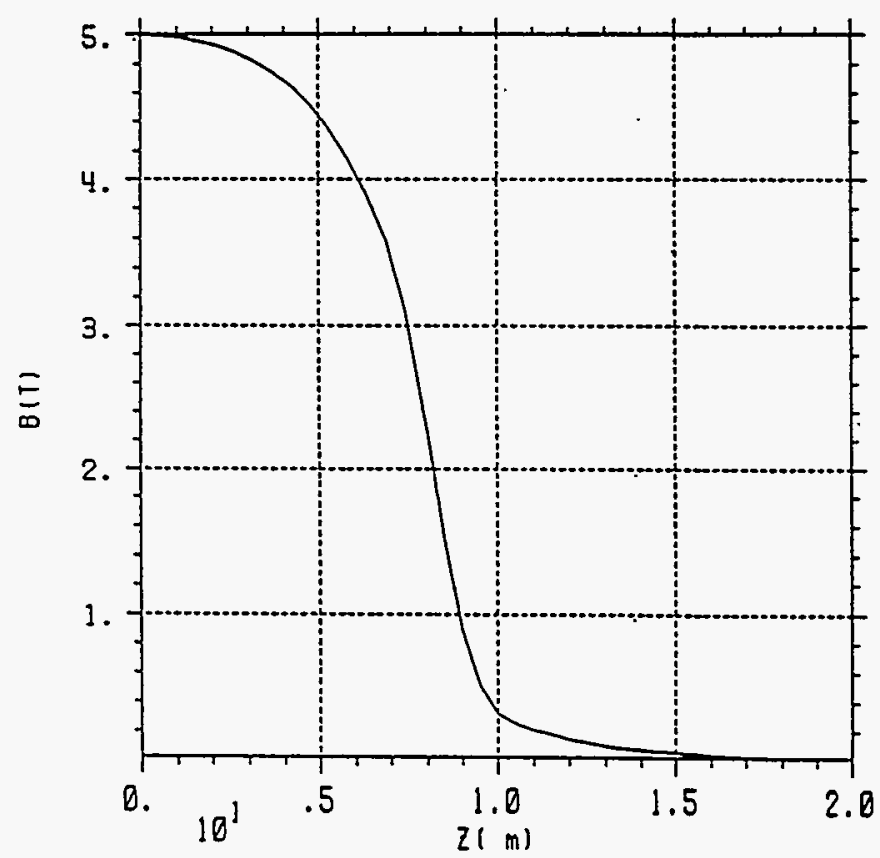

Figure 26: The magnetic field as a function of vertical distance from the beamline. (The scale is in units of $10 \mathrm{~m}$.) 
2. The total size of the detector is considerably smaller than the planned experimental hall, allowing for flexibility in installation and maintenance of the detector.

3. The alignment tolerances are less stringent.

\section{Effects of the High Field on the elements of the Inner Detector}

We discuss here a few of the effects of the high field on the performance of the inner detector. For the sake of brevity we will discuss only those issues where the increased magnetic field has been thought to have a negative impact on the detector performance.

\subsection{The Effect of the Field on the Inner Tracker}

The increased magnetic field in the inner tracker causes an increase of the Lorentz angle and drift time for all drift devices. In addition, the higher field results in an increased number of low $p_{t}$ particles with closed orbits in the inner tracker, i.e., "loopers." These two effects combine to increase the occupancy in straw tube-type trackers to uncomfortable levels.

In an $0.8 \mathrm{~T}$ field, a $2 \mathrm{~mm}$ straw tube will have a drift velocity of $40 \mu \mathrm{m} / \mathrm{nsec}$. For a collection time of $50 \mathrm{nsec}$ the inner tracker will have $12 \%$ average occupancy at standard luminosity. In a $5 \mathrm{~T}$ field, the effective drift velocity is slower, about $25 \mu \mathrm{m} / \mathrm{nsec}$, causing the average occupancy to rise to $38 \%$. More seriously, $13 \%$ of the events will have an average occupancy of $63 \%$. This appears to preclude the use of straw-tubes with the 5 Tesla field.

There are, however, other likely technology choices for the inner tracker, among them silicon (strip or drift) devices and gas microstrip chambers (GMC), which can function well in the high field environment.

Silicon strip detectors necessarily have small cells in order to achieve good resolution, and drift distances of only a few hundred microns. Our simulations indicate that even in the $5 \mathrm{~T}$ field, a standard silicon strip detector will have an average occupancy of only $0.19 \%$, which should not pose a serious problem. In silicon drift devices the Lorentz angle is relatively small (20 degrees) and could, in principle, be compensated by a re-orientation of the devices, although a full mechanical design has yet to be worked out. The high field configuration will benefit from current $R \& D$ on silicon strip and silicon drift within the GEM collaboration.

Various attempts have been made to develop devices which are insensitive to looping tracks, foremost among them are the gas microstrip chambers proposed by the $L^{*}$ LOI [11] and independently at CERN by F. Udo [12]. In principle, these devices solve the three major problems of tracking in high magnetic fields at standard-to-high luminosity: large Lorentz angles, long drift times and "loopers". The Lorentz angle effect is minimized by using low mobility high drift velocity gas $\left(\mathrm{DME} / \mathrm{CO}_{2}\right)$ and drifting axially within $20^{\circ}$ of the magnetic 

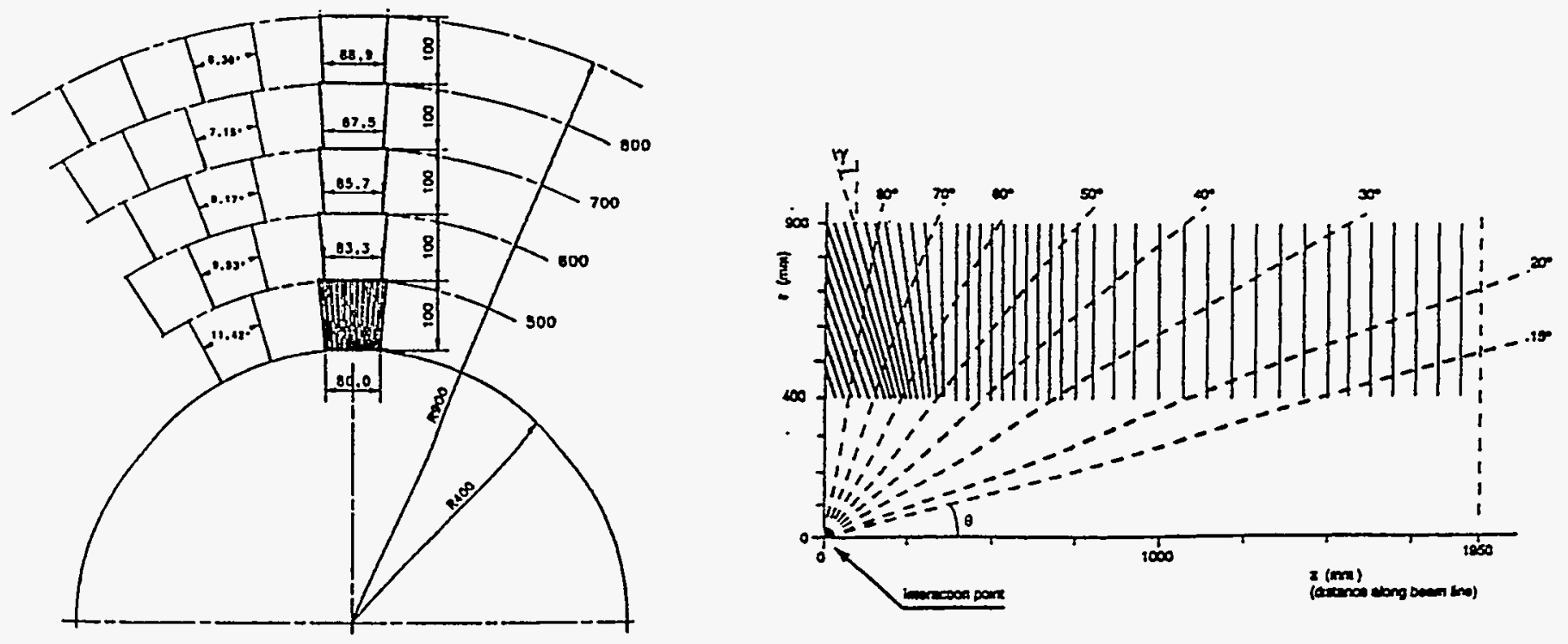

Figure 27: A possible arrangement for gas microstrip elements in the inner tracking system. Here the drift directions are always nearly parallel to the field direction and the strips are oriented radially (from ref. 11).

field direction. The drift time is minimized by using a $3 \mathrm{~mm}$ gap with a collection time of 15 to $30 \mathrm{nsec}$ depending on the incident angle. The strips can be arranged to be preferentially sensitive to radial tracks and have reduced sensitivity to wide angle, looping tracks.

A typical GMC is a 10 by $10 \mathrm{~cm}$ glass substrate ( 150 microns thick) covered by a thin metal film upon which microstrips are etched in the form of cathodes and anodes with 200 micron pitch. Tracks are incident nearly normal to the strips and pass through a $3 \mathrm{~mm}$ gas volume which is sealed by aluminized mylar. The devices are arranged so that the strips are oriented nearly radially so that high $p_{t}$ radial tracks deposit all of their ionization on one or two microstrips while large angle tracks spread their ionization over many strips, leading to a reduced pulse height on any given strip. Analog, or possibly digital (yes/no), readout of strips is possible and the discriminator threshold can be set to reject loopers. Measurements reported by F. Udo [13] showed resolutions of $\sigma=44 \mu \mathrm{m}$ and a $98 \%$ efficiency. Small angle stereo can be obtained with $1 \mathrm{~mm}$ radial resolution by tilting adjacent 10 by 10 sections by \pm 20 milliradians. An arrangement suitable for the inner tracker is shown in Fig. 27. This configuration has about 10,000 elements $(10 \mathrm{~cm}$ by $10 \mathrm{~cm}$ each) and several million readout channels. The cost of the readout can be minimized by using digital readout of the channels. The obtained resolution would comparable to the current $\mathrm{L}^{*} / \mathrm{GEM}$ inner tracker resolution.

In conclusion, the increased magnetic field may make it necessary to use either silicon or Gas Microstrip detectors as the primary elements of the inner tracker. While more R\&D is needed, there are of strong efforts in both of these areas proceeding in a number of laboratories. However, our proposed system, unlike the L*/GEM scheme, does not rely on the inner tracker for a second muon measurement, thereby alleviating some of the risks associated with the general problem of tracking at the SSC. 


\subsection{The Effect of the Field on the Endcap Region of the Middle Tracker}

The middle tracker elements in the endcap regions must function in a 5 Tesla magnetic field. These chambers are, however, shielded from the interaction point by about $14 \lambda$ of material and we estimate that the rates in this region are a rather manageable few $\mathrm{Hz} / \mathrm{cm}^{2}$. Thus, drift chambers can operate in this region without the occupancy problems that obtain in the inner tracking region.

Some of the proponents of this proposal have considerable experience with the operation of drift chambers in the $B=3$ Tesla field of the AMY detector. There we find that with care, the effects of large Lorentz angles and complex drift trajectories can be understood and spatial resolutions comparable to those obtained in the absence of a field can be acheived [14]. The AMY experiment has recently installed drift chamber arrays in the endcap regions in a configuration quite similar to that required for the end cap region of the middle tracker. Experience with the AMY endcap chambers will be directly applicable to the design and operation of the chambers required for this proposal.

\subsection{The Effect of the Field on Calorimetry}

The 5 Tesla magnetic field places constraints on the use of phototubes for light readout. However, many of these constraints are also present at the $0.8 \mathrm{~T}$ level. Cryogenic calorimetry (e.g. liquid argon and liquid xenon), which is among the final list of options being considered by GEM, can work in large magnetic fields. For example, the H1 detector at HERA has a liquid-argon calorimeter that operates in a 1.2 Tesla field. The data from their initial cosmic-ray test shows no deterioration in performance caused by the magnetic field [15]. The readout of a liquid argon calorimeter (in an "accordion" configuration) is unaffected by the field.

It has been suggested that the increased field might have a detrimental effect on the energy resolution of the electromagnetic calorimeter due to the fact the low energy shower particles initiated in the cryostat wall will curl up and vanish in the cryostat material prior to depositing their energy in the sampling medium. We are studying this effect for a cryostat with a $2 \mathrm{~cm}$ thick room temperature $\mathrm{Al}$ wall, a $1.5 \mathrm{~cm}$ vacuum space and a $2 \mathrm{~cm}$ thick cold wall. This is probably an overestimate of the material thicknesses and spacing between them and, thus, the effects on the resolution listed below are overestimates. The calorimeter is taken to be a repeating pattern of a $1.75 \mathrm{~mm} \mathrm{~Pb}$ radiator, followed by two $2 \mathrm{~mm}$ argon gaps and a $3 \mathrm{~mm}$ G-10 electrode. The mean energy deposited in argon is about $15.5 \%$.

The following table lists the values of the magnetic field, the incidence angle of the electron, the effective resolution (or the sampling term divided by the $\sqrt{E}$ ), the average and rms spread of the energy lost both due to back scatter and to the cryostat wall. The amount of backscattered energy and the amount of energy deposited in the front cryostat (dead) material increases somewhat with magnetic field, as expected, but the effect on the overall 
resolution is still not very large for normal entry. For large entry angles (60 degrees), the resolution for both the $B=0$ and $B=5 \mathrm{~T}$ cases drops by about 1.5 , which is consistent with the change expected from sampling. There are detectable, but small (order 10\%) changes in resolution when the field is turned on and off, but these are also close to the current statistical uncertainty of the EGS results. Consequently, our preliminary conclusion is that the effect of magnetic field, as such, is not very large (no more than a $15 \%$ degradation on the resolution).

\begin{tabular}{|c|c|c|c|c|c|c|c|c|c|}
\hline \multicolumn{10}{|c|}{ Electromagnetic response for a $4 \mathrm{~cm}$ thick Aluminum cryostat wall } \\
\hline \multirow{2}{*}{$\begin{array}{l}\text { Field } \\
(T)\end{array}$} & \multirow{2}{*}{$\begin{array}{l}\text { No } \\
\text { Events }\end{array}$} & \multirow{2}{*}{$\begin{array}{l}\text { Energy } \\
\text { (GeV) }\end{array}$} & \multirow{2}{*}{$\begin{array}{l}\text { Type } \\
e / \gamma\end{array}$} & \multirow{2}{*}{$\begin{array}{l}\text { Theta } \\
\text { Deg }\end{array}$} & \multirow{2}{*}{$\begin{array}{l}\text { Effective } \\
\text { Resolution }\end{array}$} & \multicolumn{2}{|c|}{ Backscatter } & \multicolumn{2}{|c|}{ Front Alum } \\
\hline & & & & & & $\%$ E lost & $R M S$ & $\%$ E lost & RMS \\
\hline 0.0 & 559 & 10 & e & 0 & 1.92 & 0.09 & 0.06 & 0.30 & 0.12 \\
\hline 0.8 & 534 & 10 & e & 0 & 1.93 & 0.09 & 0.07 & 0.30 & 0.13 \\
\hline 5.0 & 454 & 10 & $\mathbf{e}$ & 0 & 2.10 & 0.45 & 0.18 & 0.48 & 0.19 \\
\hline 0.0 & 100 & 25 & $\mathbf{e}$ & 0 & 1.29 & 0.06 & 0.02 & 0.14 & 0.07 \\
\hline 5.0 & 90 & 25 & e & 0 & 1.27 & 0.33 & 0.12 & 0.25 & 0.08 \\
\hline 0.0 & 100 & 25 & e & 60 & 1.58 & 0.51 & 0.11 & 0.70 & 0.24 \\
\hline 5.0 & 74 & 25 & e & 60 & 1.78 & 1.01 & 0.21 & 1.02 & 0.28 \\
\hline
\end{tabular}

\section{Conclusions}

In this report we present an evaluation of the capabilities of a detector based on a high field solenoid magnet. The specific scheme that is described originated as a suggestion for a highfield option for the GEM, thus, we focus the discussion on a comparison with GEM's low-field open-air muon spectrometer, which is that detector's defining characteristic. We provide preliminary results of our investigations into the questions raised by the GEM Decision Group in its memo of Sept. 10,1991, which rejected further consideration of this option. Our current evaluation of the results derived to date is that although a considerable amount of further study and optimization is needed, there are no obvious fatal deficiencies in our proposed system-the reservations raised by the GEM Decision Group are answerable in a rather straightforward manner. Moreover, the potential for significantly better momentum resolution in the inner tracker and the better $\eta$ coverage of the muon system should allow for investigations that appear to be inaccessible in the $\mathrm{L}^{*} / \mathrm{GEM}$ design.

We are encouraged that by simply matching a high-field solenoid to the $L^{*} / G E M$ configuration we find that we can improve the performance characteristics of the detector significantly. This happy situation would further improve if a proper optimization was actually carried out. Unfortunately, such an optimization would require resources that are currently unavailable to us.

Work needed to complete a Letter-of-Intent-like document includes:

1. The preparation of a conceptual design, a cost estimate, and a fabrication plan and 
schedule for the magnet coil.

2. The development of a detailed scheme for assembling and supporting the detector.

3. The complete simulation of a range of physics processes in the detector. This includes: the completion of the $p p \longrightarrow W_{L}{ }^{+} W_{L}+X$ study mentioned above; a comparative evaluation between the $L^{*} / G E M$ and high field designs' capabilities for the reactions $H \longrightarrow Z^{\circ} Z^{*} \longrightarrow l^{+} l^{-} l^{+} l^{-}$and $H \longrightarrow \gamma \gamma$; the detector's capabilities for $t$-quark physics; etc.

4. The overall optimization of the detector dimensions and a comparative study of different technologies for the various detection elements.

5. The completion of studies of $B$-field effects on calorimetry.

6. A full evaluation of an inner tracker for $B=5 \mathrm{~T}$, and the development of an R\&D plan for gas microstrip detectors.

7. A detailed cost estimate for the proposed system. 


\section{References}

[1] R.V. Steiner et al., An Expression of Interest to Construct a Major SSC Detector, SSC Laboratory EOI-0020 (1991).

[2] R. Lander, Proceedings of the International Workshop on Solenoidal Detectors for the SSC, KEK, April 1990; Winston Ko, R. Lander and P. Yager, SSC Laboratory EOI-007 (1990).

[3] J. Bensinger, E. Wang and H. Yamamoto, Proceedings of the Workshop on Computing for SSC Experiments, 1990.

[4] See, for example, M. Chanowitz and M.K. Gaillard, Nucl. Phys. B261, 379 (1985); M.S. Berger and M.S. Chanowitz, Phys. Lett. B263, 509 (1991).

[5] Report of the 1990 HEPAP Subpanel on SSC Physics, S. Drell, chairman; DOE/ER-0434 (January, 1990).

[6] V. Barger, K. Cheung, T. Han, R.J.N. Phillips, Strong $W^{+} W^{+}$Scattering Signals at $p p$ supercolliders, Preprint University of Wisconsin $\mathrm{MAD} / \mathrm{PH} / 556$. Also references within.

[7] D. Dicus, J.F. Gunion and R. Vega, Phys. Lett. B258, 475 (1991), and D. Dicus, J.F. Gunion, L.H. Orr and R. Vega, UCD-91-10 (1991).

[8] L. Osborne, presentation to the GEM Muon System Meeting, Boston University, August 23,1991 .

[9] A. Korytov, presentation to the GEM Muon System Meeting, Boston University, August 23,1991 .

[10] E. Bayham, presentation to the Workshop on Magnets for LHC Detectors, CERN, May 13, 1991.

[11] The L* collaboration LOI, SSCL 1990.

[12] F. Udo, LHC workshop, CERN 90-10, Volume III, P. 219.

[13] See for example F. Udo, CMS meeting notes, CERN CMS-Gen/91-02.

[14] K. Ueno et al., The Central Drift Chamber of the AMY Detector, to be published.

[15] G. Cozzika, private communication. 